Supplementary Information:

\title{
Absolute Configuration of (-)-Centratherin, a Sesquiterpenoid Lactone, Defined by Means of Chiroptical Spectroscopy
}

Fernando M. S. Junior, ${ }^{\dagger}+$ Cody L. Covington, ${ }^{\ddagger}$ Ana Carolina F. de Albuquerque, ${ }^{\dagger}$ Jonathas F. R. Lobo,$^{\dagger}$ Ricardo M. Borges, ${ }^{\dagger}$ Mauro B. de Amorim,${ }^{\dagger}$ and Prasad L. Polavarapu,,+ 


\section{Table of Contents}

Table S1. Theoretical $\left(\delta_{\text {scal }}\right)$ and experimental $\left(\delta_{\text {exp }}\right){ }^{13} \mathrm{C}$ NMR chemical shifts for centratherin ..Page 3

Table S2. Theoretical $\left(\delta_{\text {scal }}\right){ }^{13} \mathrm{C}$ NMR chemical shifts for budlein A and experimental $\left(\delta_{\text {exp }}\right)$ data for centratherin Page 4

Table S3: Relative energies and Boltzmann weights for the 16 lowest energy conformers of (-)$(6 R, 7 R, 8 S, 10 R, 2 ' Z)$-centratherin Page 5

Table S4: Cartesian Coordinates for the sixteen low energy conformers of (-)-(6R,7R,8S,10R,2'Z)centratherin. Page 6

Table S5: Experimental and Boltzmann population weighted $[\alpha]$ values of (-)-(6R,7R,8S,10R,2'Z)centratherin Page 23

Table S6: Vibrational frequencies $\left(\mathrm{cm}^{-1}\right)$, rotational $\left(10^{-44} \mathrm{esu}^{2} \mathrm{~cm}^{2}\right)$ and dipole strengths $\left(10^{-40} \mathrm{esu}^{2} \mathrm{~cm}^{2}\right)$ for the four lowest energy conformers of $(-)-(6 R, 7 R, 8 S, 10 R, 2 ' Z)$-centratherin. $\quad$.......Page 24

Table S7: Electronic dipole strengths and Rotational Strengths $(R)$ of four lowest energy conformers of ()-(6R,7R,8S,10R,2'Z)-centratherin. Page 31

Table S8: ORD values at camb3lyp/Aug-cc-pVDZ/PCM for 4 lowest energy conformations of (-)$(6 R, 7 R, 8 S, 10 R, 2 ' Z)$-centratherin. Page 38

Figure S1: Three dimensional structural views of the four lowest energy conformers of (-)$\left(6 R, 7 R, 8 S, 10 R, 2^{\prime} Z\right)$-centratherin. Page 39

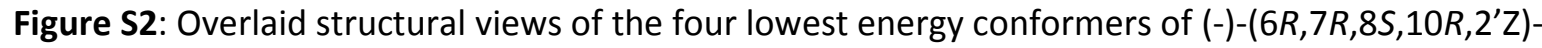
centratherin Page 40

Figure S3: Vibrational Infrared absorption spectra of four lowest energy conformers of (-)$(6 R, 7 R, 8 S, 10 R, 2 ' Z)$-centratherin Page 41

Figure S4: Vibrational circular dichroism spectra of four lowest energy conformers of (-)$(6 R, 7 R, 8 S, 10 R, 2 ' Z)$-centratherin Page 42

Figure S5: Electronic absorption spectra of four lowest energy conformers of (-)-(6R,7R,8S,10R,2'Z)centratherin Page 43

Figure S6: Electronic circular dichroism spectra of four lowest energy conformers of (-)$(6 R, 7 R, 8 S, 10 R, 2 ' Z)$-centratherin Page 44

Figure S7: Predicted electronic circular dichroism spectrum (-)-(6R,7R,8S,10R,2'Z)-centratherin (Figure 1B in the main text) with rotational strengths displayed as vertical bars and its comparison to experimental spectrum (red trace). Page 45 
Table S1. Theoretical $\left(\delta_{\text {scal }}\right)$ and experimental $\left(\delta_{\exp }\right){ }^{13} \mathrm{C}$ NMR chemical shifts for centratherin

\begin{tabular}{|c|c|c|c|}
\hline Nuclei & $\delta_{\text {scal }}$ & $\delta_{\text {exp }}$ & $\left|\delta_{\exp }-\delta_{\text {scal }}\right|$ \\
\hline 1 & 201.1 & 205.2 & 4.1 \\
\hline 2 & 106.4 & 106.8 & 0.4 \\
\hline 3 & 187.9 & 184.9 & 3.0 \\
\hline 4 & 136.6 & 135.1 & 1.5 \\
\hline 5 & 142.9 & 134.8 & 8.1 \\
\hline 6 & 81.3 & 82.1 & 0.8 \\
\hline 7 & 51.0 & 51.1 & 0.1 \\
\hline 8 & 73.1 & 73.2 & 0.1 \\
\hline 9 & 44.6 & 44.2 & 0.4 \\
\hline 10 & 89.4 & 90.1 & 0.7 \\
\hline 11 & 135.4 & 133.6 & 1.8 \\
\hline 12 & 165.6 & 169.3 & 3.7 \\
\hline 13 & 127.1 & 124.8 & 2.3 \\
\hline 14 & 21.5 & 20.9 & 0.6 \\
\hline 15 & 66.7 & 63.1 & 3.6 \\
\hline $1^{\prime}$ & 166.5 & 167.4 & 0.9 \\
\hline $2^{\prime}$ & 126.1 & 135.1 & 9.0 \\
\hline $2^{\prime}$ & 151.3 & 141.1 & 10.2 \\
\hline $4^{\prime}$ & 16.6 & 15.9 & 0.7 \\
\hline $5^{\prime}$ & 21.9 & 20.2 & 1.7 \\
\hline MAD & $\begin{array}{l}-- \\
\end{array}$ & --- & 2.68 \\
\hline RMSD & --- & --- & 3.99 \\
\hline
\end{tabular}


Table S2. Theoretical $\left(\delta_{\text {scal }}\right){ }^{13} \mathrm{C}$ NMR chemical shifts for budlein A and experimental $\left(\delta_{\text {exp }}\right)$ data for centratherin

\begin{tabular}{|c|c|c|c|}
\hline Nuclei & $\delta_{\text {scal }}$ & $\delta_{\text {exp }}$ & $\left|\delta_{\text {exp }}-\delta_{\text {scal }}\right|$ \\
\hline 1 & 203.0 & 205.2 & 2.2 \\
\hline 2 & 102.3 & 106.8 & 4.5 \\
\hline 3 & 181.9 & 184.9 & 3.0 \\
\hline 4 & 132.3 & 135.1 & 2.8 \\
\hline 5 & 147.9 & 134.8 & 13.1 \\
\hline 6 & 77.2 & 82.1 & 4.9 \\
\hline 7 & 55.2 & 51.1 & 4.1 \\
\hline 8 & 78.3 & 73.2 & 5.1 \\
\hline 9 & 39.4 & 44.2 & 4.8 \\
\hline 10 & 87.5 & 90.1 & 2.6 \\
\hline 11 & 141.7 & 133.6 & 8.1 \\
\hline 12 & 165.8 & 169.3 & 3.5 \\
\hline 13 & 127.7 & 124.8 & 2.9 \\
\hline 14 & 22.2 & 20.9 & 1.3 \\
\hline 15 & 64.1 & 63.1 & 1.0 \\
\hline $1^{\prime}$ & 164.9 & 167.4 & 2.5 \\
\hline $2^{\prime}$ & 126.6 & 135.1 & 8.5 \\
\hline $2^{\prime}$ & 151.8 & 141.1 & 10.7 \\
\hline $4^{\prime}$ & 17.0 & 15.9 & 1.1 \\
\hline $5^{\prime}$ & 22.8 & 20.2 & 2.6 \\
\hline MAD & --- & --- & 4.47 \\
\hline RMSD & --- & --- & 5.48 \\
\hline
\end{tabular}


Table S3: Relative energies and Boltzmann weights for the 16 lowest energy conformers of (-)$(6 R, 7 R, 8 S, 10 R, 2 ' Z)$-centratherin.

\begin{tabular}{|c|c|c|c|c|}
\hline $\begin{array}{l}\text { Conformation } \\
\text { Number }\end{array}$ & $\begin{array}{l}\text { Gibbs } \Delta \mathrm{E} \\
\text { (kcal/mol) }\end{array}$ & $\begin{array}{l}\text { Gibbs } \Delta E \\
\text { Populations }\end{array}$ & $\begin{array}{l}\text { Electronic } \Delta E \\
\text { (kcal/mol) }\end{array}$ & $\begin{array}{l}\text { Electronic } \Delta \mathrm{E} \\
\text { Populations }\end{array}$ \\
\hline 1 & 0.000 & 0.214 & 0.00 & 0.252 \\
\hline 2 & 0.133 & 0.171 & 0.26 & 0.162 \\
\hline 3 & 0.418 & 0.105 & 0.44 & 0.119 \\
\hline 4 & 0.427 & 0.103 & 0.47 & 0.112 \\
\hline 5 & 0.579 & 0.080 & 0.66 & 0.082 \\
\hline 6 & 0.742 & 0.060 & 1.14 & 0.036 \\
\hline 7 & 0.744 & 0.060 & 0.96 & 0.049 \\
\hline 8 & 0.789 & 0.056 & 0.95 & 0.050 \\
\hline 9 & 1.004 & 0.039 & 1.17 & 0.034 \\
\hline 10 & 1.313 & 0.023 & 1.69 & 0.014 \\
\hline 11 & 1.362 & 0.021 & 1.31 & 0.027 \\
\hline 12 & 1.399 & 0.020 & 1.66 & 0.015 \\
\hline 13 & 1.485 & 0.017 & 1.81 & 0.012 \\
\hline 14 & 1.704 & 0.012 & 1.81 & 0.012 \\
\hline 15 & 1.828 & 0.009 & 1.76 & 0.012 \\
\hline 16 & 1.830 & 0.009 & 1.76 & 0.012 \\
\hline
\end{tabular}


Table S4: Cartesian Coordinates for the sixteen low energy conformers of (-)-(6R,7R,8S,10R,2'Z)centratherin.

\begin{tabular}{|c|c|c|c|}
\hline C & 1.169361 & 1.459762 & 1.307805 \\
\hline C & 1.337471 & 2.740831 & 0.572611 \\
\hline 0 & 0.389498 & 2.835633 & -0.389824 \\
\hline $\mathrm{O}$ & 2.151514 & 3.624231 & 0.748908 \\
\hline C & 1.933761 & 1.155811 & 2.362439 \\
\hline C & -2.974913 & 1.333355 & -0.606561 \\
\hline C & -2.799482 & -0.134093 & -0.528182 \\
\hline C & -1.884006 & 2.112220 & -0.511301 \\
\hline C & -0.511946 & -1.858916 & 0.975493 \\
\hline C & 0.291875 & -0.775440 & 0.233003 \\
\hline C & -4.365239 & 1.892213 & -0.795807 \\
\hline 0 & -5.264517 & 1.488638 & 0.244116 \\
\hline 0 & -2.553717 & -0.600106 & 0.717939 \\
\hline C & -2.694827 & -1.070810 & -1.512676 \\
\hline C & -2.201788 & -2.285565 & -0.896064 \\
\hline 0 & -1.916188 & -3.368583 & -1.401340 \\
\hline 0 & 1.672907 & -1.164560 & 0.421953 \\
\hline C & 2.576501 & -0.679322 & -0.468585 \\
\hline C & 3.936453 & -1.224270 & -0.213717 \\
\hline C & 4.045269 & -2.435823 & 0.685528 \\
\hline C & 5.033979 & -0.655154 & -0.760474 \\
\hline C & 5.162964 & 0.554787 & -1.633354 \\
\hline 0 & 2.248773 & 0.093079 & -1.357712 \\
\hline $\mathrm{H}$ & -2.007757 & 3.195530 & -0.562088 \\
\hline $\mathrm{H}$ & 0.095852 & -0.832438 & -0.842515 \\
\hline $\mathrm{H}$ & 5.983846 & -1.141772 & -0.518715 \\
\hline $\mathrm{H}$ & 1.808688 & 0.234959 & 2.928002 \\
\hline $\mathrm{H}$ & 2.715788 & 1.844404 & 2.680317 \\
\hline $\mathrm{H}$ & -0.429998 & -1.708242 & 2.059881 \\
\hline $\mathrm{H}$ & -0.062361 & -2.831488 & 0.740724 \\
\hline $\mathrm{H}$ & -4.310252 & 2.987600 & -0.873258 \\
\hline $\mathrm{H}$ & -4.803822 & 1.501592 & -1.723605 \\
\hline $\mathrm{H}$ & -4.957498 & 1.870471 & 1.077582 \\
\hline $\mathrm{H}$ & -2.855176 & -0.908354 & -2.572700 \\
\hline $\mathrm{H}$ & 3.695371 & -2.212389 & 1.702710 \\
\hline $\mathrm{H}$ & 3.432874 & -3.268169 & 0.311116 \\
\hline $\mathrm{H}$ & 5.087313 & -2.770765 & 0.743932 \\
\hline $\mathrm{H}$ & 5.354134 & 0.246895 & -2.674551 \\
\hline $\mathrm{H}$ & 4.270492 & 1.184404 & -1.627542 \\
\hline $\mathrm{H}$ & 6.037006 & 1.142308 & -1.318346 \\
\hline C & -0.445108 & 1.642312 & -0.465493 \\
\hline $\mathrm{H}$ & -0.188585 & 1.172848 & -1.424029 \\
\hline C & 0.009933 & 0.700961 & 0.707026 \\
\hline $\mathrm{H}$ & -0.788799 & 0.657701 & 1.450954 \\
\hline
\end{tabular}




\begin{tabular}{|c|c|c|c|}
\hline C & -2.004569 & -1.953632 & 0.600052 \\
\hline C & -2.781317 & -2.892576 & 1.510433 \\
\hline $\mathrm{H}$ & -2.670730 & -2.590198 & 2.558615 \\
\hline $\mathrm{H}$ & -2.393225 & -3.911258 & 1.391441 \\
\hline $\mathrm{H}$ & -3.845548 & -2.890196 & 1.246696 \\
\hline \multicolumn{4}{|c|}{--- Conformation 2 - } \\
\hline C & -1.230124 & -1.391540 & 1.370446 \\
\hline C & -1.354967 & -2.691854 & 0.660876 \\
\hline $\mathrm{O}$ & -0.343285 & -2.817097 & -0.231088 \\
\hline $\mathrm{O}$ & -2.184830 & -3.566410 & 0.803935 \\
\hline C & -2.052642 & -1.060229 & 2.371681 \\
\hline C & 3.036662 & -1.339059 & -0.169503 \\
\hline C & 2.837651 & 0.126157 & -0.249611 \\
\hline C & 1.942480 & -2.117055 & -0.134714 \\
\hline C & 0.448270 & 1.933188 & 0.963900 \\
\hline C & -0.309277 & 0.800148 & 0.246433 \\
\hline C & 4.440454 & -1.895229 & -0.097245 \\
\hline $\mathrm{O}$ & 5.199117 & -1.607873 & -1.278563 \\
\hline $\mathrm{O}$ & 2.525258 & 0.701308 & 0.936714 \\
\hline C & 2.761936 & 0.964032 & -1.320710 \\
\hline C & 2.228692 & 2.223888 & -0.842858 \\
\hline $\mathrm{O}$ & 1.959961 & 3.252561 & -1.458426 \\
\hline 0 & -1.700764 & 1.184291 & 0.347500 \\
\hline C & -2.553950 & 0.643895 & -0.561103 \\
\hline C & -3.930627 & 1.183106 & -0.401917 \\
\hline C & -4.095454 & 2.446821 & 0.413006 \\
\hline C & -4.994639 & 0.562955 & -0.959093 \\
\hline C & -5.067260 & -0.702491 & -1.756619 \\
\hline $\mathrm{O}$ & -2.174640 & -0.167707 & -1.392864 \\
\hline $\mathrm{H}$ & 2.063621 & -3.198932 & -0.056364 \\
\hline $\mathrm{H}$ & -0.062855 & 0.797899 & -0.819957 \\
\hline $\mathrm{H}$ & -5.960932 & 1.048281 & -0.791164 \\
\hline $\mathrm{H}$ & -1.958112 & -0.123869 & 2.917585 \\
\hline $\mathrm{H}$ & -2.852050 & -1.740139 & 2.664006 \\
\hline $\mathrm{H}$ & 0.308367 & 1.847629 & 2.049452 \\
\hline $\mathrm{H}$ & 0.004570 & 2.884672 & 0.645889 \\
\hline $\mathrm{H}$ & 4.996214 & -1.426328 & 0.725068 \\
\hline $\mathrm{H}$ & 4.390536 & -2.977723 & 0.089309 \\
\hline $\mathrm{H}$ & 4.784585 & -2.064243 & -2.023353 \\
\hline $\mathrm{H}$ & 2.976979 & 0.706881 & -2.351588 \\
\hline $\mathrm{H}$ & -3.793286 & 2.292030 & 1.457873 \\
\hline $\mathrm{H}$ & -3.474271 & 3.262348 & 0.016670 \\
\hline $\mathrm{H}$ & -5.142547 & 2.770730 & 0.400108 \\
\hline $\mathrm{H}$ & -5.213239 & -0.465501 & -2.823338 \\
\hline $\mathrm{H}$ & -4.168063 & -1.316413 & -1.669050 \\
\hline $\mathrm{H}$ & -5.947353 & -1.282396 & -1.444437 \\
\hline C & 0.512093 & -1.638044 & -0.268383 \\
\hline $\mathrm{H}$ & 0.364413 & -1.205809 & -1.266050 \\
\hline
\end{tabular}




\begin{tabular}{|c|c|c|c|}
\hline C & -0.040105 & -0.644808 & 0.817386 \\
\hline $\mathrm{H}$ & 0.701728 & -0.547280 & 1.613529 \\
\hline C & 1.958540 & 2.025668 & 0.665158 \\
\hline C & 2.665866 & 3.060522 & 1.526503 \\
\hline $\mathrm{H}$ & 2.498219 & 2.855016 & 2.590506 \\
\hline $\mathrm{H}$ & 2.271601 & 4.056062 & 1.290201 \\
\hline $\mathrm{H}$ & 3.743758 & 3.054074 & 1.325650 \\
\hline \multicolumn{4}{|c|}{ Confor } \\
\hline C & -1.077056 & -1.411958 & 1.360287 \\
\hline C & -1.138103 & -2.718988 & 0.654458 \\
\hline 0 & -0.153374 & -2.776880 & -0.272171 \\
\hline 0 & -1.901950 & -3.647366 & 0.826915 \\
\hline C & -1.887797 & -1.132931 & 2.386703 \\
\hline C & 3.114699 & -1.071635 & -0.367342 \\
\hline C & 2.825161 & 0.378903 & -0.392606 \\
\hline C & 2.079656 & -1.921009 & -0.288929 \\
\hline C & 0.374131 & 2.009763 & 0.940226 \\
\hline C & -0.330795 & 0.840430 & 0.227396 \\
\hline C & 4.570760 & -1.493765 & -0.411524 \\
\hline 0 & 4.765976 & -2.898975 & -0.420529 \\
\hline 0 & 2.515310 & 0.902008 & 0.817197 \\
\hline C & 2.676584 & 1.241905 & -1.436448 \\
\hline C & 2.081995 & 2.453657 & -0.908007 \\
\hline $\mathrm{O}$ & 1.735118 & 3.480168 & -1.486613 \\
\hline 0 & -1.740345 & 1.136316 & 0.370632 \\
\hline C & -2.584578 & 0.552507 & -0.518971 \\
\hline C & -3.986599 & 1.004247 & -0.313922 \\
\hline C & -4.205121 & 2.238351 & 0.533170 \\
\hline C & -5.026425 & 0.334281 & -0.859078 \\
\hline C & -5.046045 & -0.915060 & -1.684542 \\
\hline $\mathrm{O}$ & -2.180288 & -0.225884 & -1.370519 \\
\hline $\mathrm{H}$ & 2.281201 & -2.991753 & -0.250854 \\
\hline $\mathrm{H}$ & -0.114070 & 0.867130 & -0.845293 \\
\hline $\mathrm{H}$ & -6.014726 & 0.758087 & -0.656324 \\
\hline $\mathrm{H}$ & -1.837517 & -0.190784 & 2.928507 \\
\hline $\mathrm{H}$ & -2.631187 & -1.863039 & 2.704612 \\
\hline $\mathrm{H}$ & 0.271663 & 1.900605 & 2.027906 \\
\hline $\mathrm{H}$ & -0.136582 & 2.936081 & 0.649846 \\
\hline $\mathrm{H}$ & 5.052661 & -1.017605 & -1.281339 \\
\hline $\mathrm{H}$ & 5.085525 & -1.116366 & 0.483789 \\
\hline $\mathrm{H}$ & 4.506275 & -3.245187 & -1.284949 \\
\hline $\mathrm{H}$ & 2.875781 & 1.026402 & -2.480340 \\
\hline $\mathrm{H}$ & -3.864665 & 2.080833 & 1.565786 \\
\hline $\mathrm{H}$ & -3.646029 & 3.098462 & 0.138641 \\
\hline $\mathrm{H}$ & -5.269928 & 2.497555 & 0.555717 \\
\hline $\mathrm{H}$ & -5.233803 & -0.663103 & -2.741272 \\
\hline $\mathrm{H}$ & -4.110566 & -1.476588 & -1.634064 \\
\hline $\mathrm{H}$ & -5.882302 & -1.552704 & -1.364487 \\
\hline
\end{tabular}




\begin{tabular}{|c|c|c|c|}
\hline C & 0.615381 & -1.538863 & -0.345089 \\
\hline $\mathrm{H}$ & 0.383453 & -1.116726 & -1.331062 \\
\hline C & 0.044347 & -0.591019 & 0.770565 \\
\hline $\mathrm{H}$ & 0.808227 & -0.457520 & 1.540024 \\
\hline C & 1.866746 & 2.198401 & 0.600538 \\
\hline C & 2.540241 & 3.248149 & 1.471112 \\
\hline $\mathrm{H}$ & 2.418521 & 3.002788 & 2.532877 \\
\hline $\mathrm{H}$ & 2.081590 & 4.225124 & 1.277156 \\
\hline $\mathrm{H}$ & 3.609787 & 3.311002 & 1.237833 \\
\hline \multicolumn{4}{|c|}{--- Conformati } \\
\hline C & 1.063844 & 1.430931 & 1.337833 \\
\hline C & 1.130582 & 2.730261 & 0.618360 \\
\hline 0 & 0.155910 & 2.776474 & -0.319295 \\
\hline $\mathrm{O}$ & 1.891069 & 3.661723 & 0.789193 \\
\hline C & 1.865233 & 1.164096 & 2.374793 \\
\hline C & -3.109203 & 1.065847 & -0.419135 \\
\hline C & -2.818922 & -0.384849 & -0.421742 \\
\hline C & -2.075437 & 1.917520 & -0.352966 \\
\hline C & -0.380951 & -1.995786 & 0.951785 \\
\hline C & 0.329021 & -0.836376 & 0.227405 \\
\hline C & -4.565923 & 1.484742 & -0.474398 \\
\hline $\mathrm{O}$ & -4.766466 & 2.888307 & -0.422114 \\
\hline $\mathrm{O}$ & -2.522918 & -0.893158 & 0.797883 \\
\hline C & -2.659544 & -1.260548 & -1.453178 \\
\hline C & -2.072605 & -2.466500 & -0.903252 \\
\hline $\mathrm{O}$ & -1.722067 & -3.501111 & -1.464881 \\
\hline $\mathrm{O}$ & 1.737378 & -1.129550 & 0.387155 \\
\hline C & 2.589489 & -0.557962 & -0.502894 \\
\hline C & 3.989630 & -1.006598 & -0.278948 \\
\hline C & 4.200827 & -2.226475 & 0.590342 \\
\hline C & 5.034197 & -0.346316 & -0.826902 \\
\hline C & 5.061283 & 0.888719 & -1.673399 \\
\hline 0 & 2.192703 & 0.208446 & -1.368677 \\
\hline $\mathrm{H}$ & -2.277926 & 2.988747 & -0.350818 \\
\hline $\mathrm{H}$ & 0.121420 & -0.878853 & -0.846552 \\
\hline $\mathrm{H}$ & 6.020567 & -0.767145 & -0.609096 \\
\hline $\mathrm{H}$ & 1.810880 & 0.228059 & 2.926637 \\
\hline $\mathrm{H}$ & 2.605019 & 1.898463 & 2.691292 \\
\hline $\mathrm{H}$ & -0.287015 & -1.870774 & 2.038522 \\
\hline $\mathrm{H}$ & 0.132936 & -2.925694 & 0.679005 \\
\hline $\mathrm{H}$ & -4.999762 & 1.144384 & -1.425599 \\
\hline $\mathrm{H}$ & -5.119777 & 0.973604 & 0.330278 \\
\hline $\mathrm{H}$ & -4.593777 & 3.195471 & 0.477939 \\
\hline $\mathrm{H}$ & -2.847312 & -1.057891 & -2.501742 \\
\hline $\mathrm{H}$ & 3.851348 & -2.052006 & 1.617210 \\
\hline $\mathrm{H}$ & 3.645266 & -3.093117 & 0.205222 \\
\hline $\mathrm{H}$ & 5.265419 & -2.485024 & 0.626498 \\
\hline $\mathrm{H}$ & 5.257170 & 0.618530 & -2.724139 \\
\hline
\end{tabular}




\begin{tabular}{|c|c|c|c|}
\hline $\mathrm{H}$ & 4.125857 & 1.451578 & -1.639954 \\
\hline $\mathrm{H}$ & 5.895512 & 1.531195 & -1.357747 \\
\hline C & -0.610145 & 1.536263 & -0.387265 \\
\hline $\mathrm{H}$ & -0.365592 & 1.103555 & -1.365662 \\
\hline C & -0.051347 & 0.602636 & 0.746427 \\
\hline $\mathrm{H}$ & -0.823561 & 0.479605 & 1.509319 \\
\hline C & -1.870751 & -2.191444 & 0.603570 \\
\hline C & -2.549351 & -3.230443 & 1.482820 \\
\hline $\mathrm{H}$ & -2.435320 & -2.971399 & 2.542202 \\
\hline $\mathrm{H}$ & -2.088601 & -4.209440 & 1.304701 \\
\hline $\mathrm{H}$ & -3.617189 & -3.297214 & 1.242839 \\
\hline \multicolumn{4}{|c|}{ onform } \\
\hline C & 1.083524 & 1.717141 & 1.171996 \\
\hline C & 1.050985 & 3.015051 & 0.447975 \\
\hline 0 & 0.047325 & 2.998504 & -0.461160 \\
\hline 0 & 1.757616 & 3.991544 & 0.593642 \\
\hline C & 1.934707 & 1.500300 & 2.180522 \\
\hline C & -3.112731 & 1.090617 & -0.508762 \\
\hline C & -2.754365 & -0.344280 & -0.455194 \\
\hline C & -2.122788 & 1.998548 & -0.468808 \\
\hline C & -0.185562 & -1.779587 & 0.890665 \\
\hline C & 0.431056 & -0.601763 & 0.113517 \\
\hline C & -4.569931 & 1.473935 & -0.613290 \\
\hline $\mathrm{O}$ & -5.349834 & 0.965762 & 0.475975 \\
\hline $\mathrm{O}$ & -2.379256 & -0.780169 & 0.769530 \\
\hline C & -2.595810 & -1.258710 & -1.453369 \\
\hline C & -1.921906 & -2.405425 & -0.879156 \\
\hline $\mathrm{O}$ & -1.536947 & -3.444017 & -1.410984 \\
\hline $\mathrm{O}$ & 1.862528 & -0.814098 & 0.216675 \\
\hline C & 2.635494 & -0.209947 & -0.715534 \\
\hline C & 4.092571 & -0.490481 & -0.607179 \\
\hline C & 4.959094 & 0.528837 & -1.313592 \\
\hline C & 4.636356 & -1.563689 & 0.007965 \\
\hline C & 4.005765 & -2.731731 & 0.703322 \\
\hline $\mathrm{O}$ & 2.160856 & 0.537886 & -1.559599 \\
\hline $\mathrm{H}$ & -2.382847 & 3.058182 & -0.502933 \\
\hline $\mathrm{H}$ & 0.178801 & -0.679128 & -0.948614 \\
\hline $\mathrm{H}$ & 5.729480 & -1.606584 & -0.021786 \\
\hline $\mathrm{H}$ & 1.953637 & 0.564953 & 2.735606 \\
\hline $\mathrm{H}$ & 2.641935 & 2.276893 & 2.469358 \\
\hline $\mathrm{H}$ & -0.054251 & -1.626201 & 1.969742 \\
\hline $\mathrm{H}$ & 0.360980 & -2.689699 & 0.615962 \\
\hline $\mathrm{H}$ & -4.654263 & 2.567538 & -0.688568 \\
\hline $\mathrm{H}$ & -5.011371 & 1.030139 & -1.515406 \\
\hline $\mathrm{H}$ & -5.042203 & 1.384040 & 1.291502 \\
\hline $\mathrm{H}$ & -2.838191 & -1.114597 & -2.500356 \\
\hline $\mathrm{H}$ & 4.746862 & 0.546792 & -2.391575 \\
\hline $\mathrm{H}$ & 4.769342 & 1.543037 & -0.934577 \\
\hline
\end{tabular}




\begin{tabular}{|c|c|c|c|}
\hline $\mathrm{H}$ & 6.019892 & 0.293866 & -1.170591 \\
\hline $\mathrm{H}$ & 4.533876 & -3.651966 & 0.416484 \\
\hline H & 4.128486 & -2.631320 & 1.793985 \\
\hline $\mathrm{H}$ & 2.941562 & -2.845881 & 0.489319 \\
\hline C & -0.636451 & 1.711404 & -0.509249 \\
\hline $\mathrm{H}$ & -0.378325 & 1.285149 & -1.487266 \\
\hline C & -0.003537 & 0.824845 & 0.622066 \\
\hline $\mathrm{H}$ & -0.748008 & 0.676977 & 1.407463 \\
\hline C & -1.676203 & -2.055145 & 0.605711 \\
\hline C & -2.273800 & -3.084999 & 1.552273 \\
\hline $\mathrm{H}$ & -2.137343 & -2.773672 & 2.594771 \\
\hline $\mathrm{H}$ & -1.770839 & -4.047496 & 1.400083 \\
\hline $\mathrm{H}$ & -3.344322 & -3.213844 & 1.353498 \\
\hline \multicolumn{4}{|c|}{50} \\
\hline C & -0.934676 & -1.716031 & 1.196591 \\
\hline C & -0.756806 & -3.003019 & 0.473658 \\
\hline $\mathrm{O}$ & 0.276719 & -2.893524 & -0.393398 \\
\hline $\mathrm{O}$ & -1.379310 & -4.039226 & 0.590530 \\
\hline C & -1.827959 & -1.586499 & 2.183561 \\
\hline C & 3.248925 & -0.715835 & -0.259762 \\
\hline C & 2.740800 & 0.672625 & -0.308609 \\
\hline C & 2.352567 & -1.713499 & -0.249645 \\
\hline C & -0.012082 & 1.898740 & 0.865500 \\
\hline C & -0.487387 & 0.639923 & 0.115733 \\
\hline C & 4.751964 & -0.909549 & -0.203045 \\
\hline $\mathrm{O}$ & 5.160320 & -2.268130 & -0.203601 \\
\hline 0 & 2.278832 & 1.134099 & 0.877639 \\
\hline C & 2.523782 & 1.507312 & -1.363352 \\
\hline C & 1.715924 & 2.608526 & -0.877727 \\
\hline $\mathrm{O}$ & 1.248122 & 3.570861 & -1.480894 \\
\hline $\mathrm{O}$ & -1.934721 & 0.709289 & 0.186463 \\
\hline C & -2.622044 & 0.015970 & -0.751019 \\
\hline C & -4.102336 & 0.145742 & -0.676606 \\
\hline C & -4.842996 & -0.956720 & -1.401225 \\
\hline C & -4.768949 & 1.155633 & -0.075048 \\
\hline C & -4.281331 & 2.381315 & 0.635648 \\
\hline $\mathrm{O}$ & -2.055237 & -0.689691 & -1.574194 \\
\hline $\mathrm{H}$ & 2.710522 & -2.741827 & -0.195061 \\
\hline $\mathrm{H}$ & -0.221430 & 0.710414 & -0.943425 \\
\hline $\mathrm{H}$ & -5.859596 & 1.084248 & -0.130795 \\
\hline $\mathrm{H}$ & -1.949790 & -0.660319 & 2.741423 \\
\hline $\mathrm{H}$ & -2.464874 & -2.428446 & 2.452408 \\
\hline $\mathrm{H}$ & -0.163715 & 1.772193 & 1.945363 \\
\hline $\mathrm{H}$ & -0.636139 & 2.739563 & 0.539467 \\
\hline $\mathrm{H}$ & 5.213800 & -0.352721 & -1.035059 \\
\hline $\mathrm{H}$ & 5.139761 & -0.470781 & 0.727636 \\
\hline $\mathrm{H}$ & 5.008539 & -2.639360 & -1.083263 \\
\hline $\mathrm{H}$ & 2.817940 & 1.331025 & -2.392047 \\
\hline
\end{tabular}




\begin{tabular}{|c|c|c|c|}
\hline $\mathrm{H}$ & -4.608145 & -0.949808 & -2.474656 \\
\hline $\mathrm{H}$ & -4.556211 & -1.946421 & -1.018654 \\
\hline $\mathrm{H}$ & -5.925225 & -0.833850 & -1.280129 \\
\hline $\mathrm{H}$ & -4.427804 & 2.268619 & 1.722199 \\
\hline $\mathrm{H}$ & -3.228063 & 2.602278 & 0.454703 \\
\hline $\mathrm{H}$ & -4.890824 & 3.243445 & 0.330022 \\
\hline C & 0.854494 & -1.554132 & -0.404001 \\
\hline $\mathrm{H}$ & 0.628747 & -1.153554 & -1.400244 \\
\hline C & 0.075797 & -0.721241 & 0.677653 \\
\hline $\mathrm{H}$ & 0.761783 & -0.477779 & 1.492092 \\
\hline C & 1.451944 & 2.315251 & 0.616215 \\
\hline C & 1.899535 & 3.452169 & 1.522218 \\
\hline $\mathrm{H}$ & 1.752885 & 3.184094 & 2.575339 \\
\hline $\mathrm{H}$ & 1.306710 & 4.346779 & 1.297076 \\
\hline $\mathrm{H}$ & 2.958341 & 3.682703 & 1.354821 \\
\hline \multicolumn{4}{|c|}{--- Conformation 7 ----- } \\
\hline C & 1.131791 & 1.693851 & 1.212202 \\
\hline C & 1.049872 & 2.991665 & 0.491842 \\
\hline $\mathrm{O}$ & -0.014031 & 2.974916 & -0.346560 \\
\hline $\mathrm{O}$ & 1.764705 & 3.968140 & 0.588379 \\
\hline C & 2.036591 & 1.483218 & 2.174304 \\
\hline C & -3.163406 & 1.069238 & -0.090872 \\
\hline C & -2.779646 & -0.358373 & -0.175690 \\
\hline C & -2.180487 & 1.983860 & -0.123595 \\
\hline C & -0.109860 & -1.825437 & 0.906377 \\
\hline C & 0.451361 & -0.610902 & 0.143005 \\
\hline C & -4.621372 & 1.437512 & 0.064398 \\
\hline 0 & -5.407511 & 1.032015 & -1.062916 \\
\hline $\mathrm{O}$ & -2.329114 & -0.874198 & 0.993505 \\
\hline C & -2.655784 & -1.191923 & -1.245623 \\
\hline C & -1.936487 & -2.365775 & -0.793496 \\
\hline 0 & -1.570380 & -3.357989 & -1.418515 \\
\hline $\mathrm{O}$ & 1.888689 & -0.805027 & 0.170916 \\
\hline C & 2.604812 & -0.166489 & -0.783872 \\
\hline C & 4.069258 & -0.427699 & -0.756209 \\
\hline C & 4.886291 & 0.628462 & -1.467521 \\
\hline C & 4.656474 & -1.513886 & -0.207548 \\
\hline C & 4.075387 & -2.715094 & 0.474255 \\
\hline $\mathrm{O}$ & 2.077176 & 0.594634 & -1.583292 \\
\hline $\mathrm{H}$ & -2.438003 & 3.041567 & -0.043961 \\
\hline $\mathrm{H}$ & 0.150004 & -0.654899 & -0.907938 \\
\hline $\mathrm{H}$ & 5.747249 & -1.540049 & -0.292320 \\
\hline $\mathrm{H}$ & 2.089926 & 0.548229 & 2.728171 \\
\hline $\mathrm{H}$ & 2.753356 & 2.264179 & 2.425479 \\
\hline $\mathrm{H}$ & 0.080405 & -1.713654 & 1.981813 \\
\hline $\mathrm{H}$ & 0.431675 & -2.715626 & 0.564282 \\
\hline $\mathrm{H}$ & -5.057971 & 0.914224 & 0.925020 \\
\hline $\mathrm{H}$ & -4.703269 & 2.520245 & 0.237390 \\
\hline
\end{tabular}




\begin{tabular}{|c|c|c|c|}
\hline $\mathrm{H}$ & -5.106223 & 1.527140 & -1.836760 \\
\hline $\mathrm{H}$ & -2.960314 & -0.977120 & -2.263556 \\
\hline $\mathrm{H}$ & 4.700185 & 1.626205 & -1.045468 \\
\hline $\mathrm{H}$ & 5.955955 & 0.405767 & -1.382721 \\
\hline $\mathrm{H}$ & 4.622889 & 0.679076 & -2.533118 \\
\hline $\mathrm{H}$ & 3.002136 & -2.832673 & 0.314114 \\
\hline $\mathrm{H}$ & 4.595176 & -3.617529 & 0.122740 \\
\hline $\mathrm{H}$ & 4.257023 & -2.656164 & 1.559687 \\
\hline C & -0.709028 & 1.694299 & -0.335018 \\
\hline $\mathrm{H}$ & -0.559898 & 1.275658 & -1.338212 \\
\hline C & 0.024544 & 0.792030 & 0.722101 \\
\hline $\mathrm{H}$ & -0.656349 & 0.605213 & 1.555611 \\
\hline C & -1.610125 & -2.116063 & 0.695723 \\
\hline C & -2.125477 & -3.224208 & 1.601090 \\
\hline $\mathrm{H}$ & -1.925606 & -2.985877 & 2.652615 \\
\hline $\mathrm{H}$ & -1.618232 & -4.162447 & 1.346481 \\
\hline $\mathrm{H}$ & -3.204637 & -3.361098 & 1.463400 \\
\hline \multicolumn{4}{|c|}{ forn } \\
\hline C & 1.236676 & 1.395843 & 1.367708 \\
\hline C & 1.367517 & 2.695448 & 0.657810 \\
\hline $\mathrm{O}$ & 0.358851 & 2.823503 & -0.236810 \\
\hline $\mathrm{O}$ & 2.199865 & 3.567368 & 0.803007 \\
\hline C & 2.056631 & 1.062113 & 2.370251 \\
\hline C & -3.025280 & 1.355470 & -0.185635 \\
\hline C & -2.829862 & -0.110492 & -0.252430 \\
\hline C & -1.929520 & 2.130363 & -0.154224 \\
\hline C & -0.453550 & -1.922332 & 0.970370 \\
\hline C & 0.307915 & -0.794747 & 0.248062 \\
\hline C & -4.430747 & 1.912164 & -0.123133 \\
\hline $\mathrm{O}$ & -5.164323 & 1.677569 & -1.333674 \\
\hline $\mathrm{O}$ & -2.525377 & -0.682096 & 0.937299 \\
\hline C & -2.759853 & -0.954577 & -1.319408 \\
\hline C & -2.236163 & -2.216527 & -0.833463 \\
\hline $\mathrm{O}$ & -1.974798 & -3.249897 & -1.443302 \\
\hline $\mathrm{O}$ & 1.697775 & -1.184144 & 0.350643 \\
\hline C & 2.553392 & -0.651199 & -0.560213 \\
\hline C & 3.927753 & -1.195412 & -0.398114 \\
\hline C & 4.088117 & -2.451744 & 0.429044 \\
\hline C & 4.993826 & -0.586254 & -0.963512 \\
\hline C & 5.071495 & 0.670452 & -1.774231 \\
\hline $\mathrm{O}$ & 2.177447 & 0.158230 & -1.395560 \\
\hline $\mathrm{H}$ & -2.047407 & 3.213526 & -0.090854 \\
\hline $\mathrm{H}$ & 0.061402 & -0.795781 & -0.818332 \\
\hline $\mathrm{H}$ & 5.957924 & -1.074893 & -0.792576 \\
\hline $\mathrm{H}$ & 1.958172 & 0.126515 & 2.916734 \\
\hline $\mathrm{H}$ & 2.858007 & 1.739454 & 2.663156 \\
\hline $\mathrm{H}$ & -0.312868 & -1.832934 & 2.055479 \\
\hline $\mathrm{H}$ & -0.013448 & -2.876701 & 0.656052 \\
\hline
\end{tabular}




\begin{tabular}{|c|c|c|c|}
\hline $\mathrm{H}$ & -4.970793 & 1.488704 & 0.737405 \\
\hline $\mathrm{H}$ & -4.379721 & 2.999395 & 0.002022 \\
\hline $\mathrm{H}$ & -5.501737 & 0.772532 & -1.328506 \\
\hline $\mathrm{H}$ & -2.963909 & -0.699280 & -2.353123 \\
\hline $\mathrm{H}$ & 3.787157 & -2.285509 & 1.472500 \\
\hline $\mathrm{H}$ & 3.463554 & -3.268711 & 0.041091 \\
\hline $\mathrm{H}$ & 5.133936 & -2.779821 & 0.418807 \\
\hline $\mathrm{H}$ & 4.175922 & 1.290189 & -1.690886 \\
\hline $\mathrm{H}$ & 5.955613 & 1.248590 & -1.470254 \\
\hline $\mathrm{H}$ & 5.213513 & 0.421557 & -2.838785 \\
\hline C & -0.499469 & 1.646409 & -0.277272 \\
\hline $\mathrm{H}$ & -0.346439 & 1.212449 & -1.273431 \\
\hline C & 0.044481 & 0.653612 & 0.813048 \\
\hline $\mathrm{H}$ & -0.700112 & 0.562815 & 1.607409 \\
\hline C & -1.964542 & -2.010711 & 0.673315 \\
\hline C & -2.675417 & -3.037318 & 1.541471 \\
\hline $\mathrm{H}$ & -2.505857 & -2.826016 & 2.603998 \\
\hline $\mathrm{H}$ & -2.285352 & -4.035849 & 1.310905 \\
\hline $\mathrm{H}$ & -3.753483 & -3.027860 & 1.341702 \\
\hline \\
\hline C & -0.923841 & -1.722580 & 1.170927 \\
\hline C & -0.757714 & -3.003193 & 0.433958 \\
\hline $\mathrm{O}$ & 0.266113 & -2.888224 & -0.443666 \\
\hline $\mathrm{O}$ & -1.381648 & -4.038840 & 0.548402 \\
\hline C & -1.805234 & -1.600209 & 2.169436 \\
\hline C & 3.245199 & -0.720395 & -0.303660 \\
\hline C & 2.739994 & 0.669689 & -0.339988 \\
\hline C & 2.347108 & -1.716201 & -0.305864 \\
\hline C & 0.001151 & 1.893315 & 0.863074 \\
\hline C & -0.483996 & 0.642079 & 0.106630 \\
\hline C & 4.747755 & -0.916580 & -0.243810 \\
\hline 0 & 5.150443 & -2.274440 & -0.160573 \\
\hline 0 & 2.292094 & 1.127250 & 0.853338 \\
\hline C & 2.514206 & 1.509477 & -1.388650 \\
\hline C & 1.715190 & 2.611204 & -0.889469 \\
\hline $\mathrm{O}$ & 1.245369 & 3.578501 & -1.482939 \\
\hline $\mathrm{O}$ & -1.930242 & 0.712773 & 0.194879 \\
\hline C & -2.629403 & 0.029861 & -0.741585 \\
\hline C & -4.108733 & 0.158048 & -0.648677 \\
\hline C & -4.856611 & -0.923816 & -1.396693 \\
\hline C & -4.770479 & 1.148825 & -0.010747 \\
\hline C & -4.279249 & 2.353194 & 0.733023 \\
\hline $\mathrm{O}$ & -2.072536 & -0.666073 & -1.579719 \\
\hline $\mathrm{H}$ & 2.703381 & -2.746109 & -0.277636 \\
\hline $\mathrm{H}$ & -0.230030 & 0.722144 & -0.954752 \\
\hline $\mathrm{H}$ & -5.861511 & 1.076889 & -0.057939 \\
\hline $\mathrm{H}$ & -1.918308 & -0.679029 & 2.737298 \\
\hline $\mathrm{H}$ & -2.441119 & -2.443107 & 2.437730 \\
\hline
\end{tabular}




\begin{tabular}{|c|c|c|c|}
\hline $\mathrm{H}$ & -0.141368 & 1.757751 & 1.943110 \\
\hline $\mathrm{H}$ & -0.624280 & 2.737956 & 0.549667 \\
\hline $\mathrm{H}$ & 5.197823 & -0.514908 & -1.162987 \\
\hline $\mathrm{H}$ & 5.155329 & -0.328428 & 0.594965 \\
\hline $\mathrm{H}$ & 4.952448 & -2.605446 & 0.725778 \\
\hline $\mathrm{H}$ & 2.796957 & 1.337095 & -2.421176 \\
\hline $\mathrm{H}$ & -4.631291 & -0.887694 & -2.471593 \\
\hline $\mathrm{H}$ & -4.567036 & -1.923759 & -1.044057 \\
\hline $\mathrm{H}$ & -5.937632 & -0.803896 & -1.262584 \\
\hline $\mathrm{H}$ & -4.419116 & 2.207346 & 1.816536 \\
\hline $\mathrm{H}$ & -3.227478 & 2.580905 & 0.552447 \\
\hline $\mathrm{H}$ & -4.891925 & 3.223249 & 0.457649 \\
\hline C & 0.848392 & -1.550625 & -0.447289 \\
\hline $\mathrm{H}$ & 0.615449 & -1.140717 & -1.438085 \\
\hline C & 0.082909 & -0.725295 & 0.649442 \\
\hline $\mathrm{H}$ & 0.778237 & -0.490356 & 1.458437 \\
\hline C & 1.463654 & 2.310334 & 0.605026 \\
\hline C & 1.918682 & 3.441833 & 1.513978 \\
\hline $\mathrm{H}$ & 1.780186 & 3.167778 & 2.566683 \\
\hline $\mathrm{H}$ & 1.324437 & 4.337929 & 1.298727 \\
\hline $\mathrm{H}$ & 2.976227 & 3.673042 & 1.339629 \\
\hline & \multicolumn{3}{|c|}{-- Conformation 10} \\
\hline C & 1.133389 & 1.697587 & 1.204081 \\
\hline C & 1.058953 & 2.991853 & 0.476531 \\
\hline $\mathrm{O}$ & -0.001567 & 2.974398 & -0.365929 \\
\hline $\mathrm{O}$ & 1.776960 & 3.966252 & 0.570873 \\
\hline C & 2.032977 & 1.489014 & 2.171490 \\
\hline C & -3.157820 & 1.083101 & -0.104782 \\
\hline C & -2.778522 & -0.346761 & -0.185846 \\
\hline C & -2.172385 & 1.993664 & -0.143050 \\
\hline C & -0.116189 & -1.819692 & 0.906311 \\
\hline C & 0.451664 & -0.609403 & 0.141162 \\
\hline C & -4.607840 & 1.450770 & 0.069025 \\
\hline $\mathrm{O}$ & -5.322061 & 1.035631 & -1.105791 \\
\hline $\mathrm{O}$ & -2.333788 & -0.863303 & 0.985325 \\
\hline C & -2.651184 & -1.179979 & -1.255353 \\
\hline C & -1.937152 & -2.355775 & -0.800139 \\
\hline $\mathrm{O}$ & -1.571660 & -3.349445 & -1.423293 \\
\hline $\mathrm{O}$ & 1.888366 & -0.807092 & 0.176208 \\
\hline C & 2.610033 & -0.174303 & -0.778296 \\
\hline C & 4.073696 & -0.439140 & -0.743937 \\
\hline C & 4.895959 & 0.611589 & -1.457284 \\
\hline C & 4.656193 & -1.524186 & -0.188045 \\
\hline C & 4.069691 & -2.720685 & 0.497386 \\
\hline 0 & 2.087589 & 0.584922 & -1.582872 \\
\hline $\mathrm{H}$ & -2.426009 & 3.052603 & -0.071864 \\
\hline $\mathrm{H}$ & 0.154977 & -0.656326 & -0.910950 \\
\hline $\mathrm{H}$ & 5.747198 & -1.553498 & -0.268777 \\
\hline
\end{tabular}




\begin{tabular}{|c|c|c|c|}
\hline $\mathrm{H}$ & 2.080620 & 0.556700 & 2.730354 \\
\hline $\mathrm{H}$ & 2.751124 & 2.268910 & 2.422012 \\
\hline $\mathrm{H}$ & 0.070059 & -1.704980 & 1.982147 \\
\hline $\mathrm{H}$ & 0.424105 & -2.712545 & 0.569224 \\
\hline $\mathrm{H}$ & -5.016521 & 0.943519 & 0.957513 \\
\hline $\mathrm{H}$ & -4.691440 & 2.537340 & 0.216359 \\
\hline $\mathrm{H}$ & -6.266037 & 1.032199 & -0.902579 \\
\hline $\mathrm{H}$ & -2.952247 & -0.964088 & -2.274015 \\
\hline $\mathrm{H}$ & 4.636419 & 0.657890 & -2.524024 \\
\hline $\mathrm{H}$ & 4.710893 & 1.611779 & -1.040601 \\
\hline $\mathrm{H}$ & 5.964747 & 0.386587 & -1.367660 \\
\hline $\mathrm{H}$ & 4.248298 & -2.657358 & 1.583077 \\
\hline $\mathrm{H}$ & 2.996603 & -2.835982 & 0.334562 \\
\hline $\mathrm{H}$ & 4.588014 & -3.626137 & 0.151500 \\
\hline C & -0.701776 & 1.696543 & -0.349757 \\
\hline $\mathrm{H}$ & -0.552203 & 1.272823 & -1.350708 \\
\hline C & 0.025752 & 0.796598 & 0.713365 \\
\hline $\mathrm{H}$ & -0.658560 & 0.614375 & 1.545087 \\
\hline C & -1.616347 & -2.106842 & 0.690380 \\
\hline C & -2.137625 & -3.213624 & 1.593978 \\
\hline $\mathrm{H}$ & -1.939776 & -2.976428 & 2.646143 \\
\hline $\mathrm{H}$ & -1.632887 & -4.153472 & 1.340343 \\
\hline $\mathrm{H}$ & -3.216888 & -3.346778 & 1.453376 \\
\hline & \multicolumn{3}{|c|}{----- Conformation 11 ---------------- } \\
\hline C & 0.233171 & 2.695974 & 0.118442 \\
\hline C & -0.659374 & 3.544703 & -0.715019 \\
\hline $\mathrm{O}$ & -1.710366 & 2.799525 & -1.142350 \\
\hline $\mathrm{O}$ & -0.552448 & 4.715969 & -1.013029 \\
\hline C & 1.263296 & 3.217874 & 0.793431 \\
\hline C & -3.446919 & -0.022842 & 0.426815 \\
\hline C & -2.648381 & -1.194874 & 0.017998 \\
\hline C & -2.918059 & 1.188508 & 0.165340 \\
\hline C & 0.227147 & -1.020239 & -1.228838 \\
\hline C & 0.727986 & 0.318777 & -0.641593 \\
\hline C & -4.787274 & -0.217596 & 1.088606 \\
\hline $\mathrm{O}$ & -4.688257 & -0.953356 & 2.315023 \\
\hline $\mathrm{O}$ & -1.394829 & -1.165050 & 0.526318 \\
\hline C & -2.849721 & -2.184279 & -0.897483 \\
\hline C & -1.558297 & -2.807023 & -1.141599 \\
\hline $\mathrm{O}$ & -1.260119 & -3.723254 & -1.902873 \\
\hline 0 & 1.772747 & 0.079123 & 0.338553 \\
\hline C & 3.051492 & -0.059091 & -0.116559 \\
\hline C & 3.992424 & -0.345666 & 1.001075 \\
\hline C & 3.499136 & -0.127719 & 2.414594 \\
\hline C & 5.243777 & -0.799465 & 0.762918 \\
\hline C & 5.928072 & -1.123671 & -0.529151 \\
\hline $\mathrm{O}$ & 3.335733 & 0.054477 & -1.297837 \\
\hline $\mathrm{H}$ & -3.468644 & 2.086039 & 0.451920 \\
\hline
\end{tabular}




\begin{tabular}{|c|c|c|c|}
\hline $\mathrm{H}$ & 1.179793 & 0.856285 & -1.483038 \\
\hline $\mathrm{H}$ & 5.855757 & -0.972027 & 1.653517 \\
\hline $\mathrm{H}$ & 1.895719 & 2.614516 & 1.440361 \\
\hline $\mathrm{H}$ & 1.486497 & 4.280844 & 0.708580 \\
\hline $\mathrm{H}$ & 1.096561 & -1.540535 & -1.647488 \\
\hline $\mathrm{H}$ & -0.407206 & -0.773787 & -2.089691 \\
\hline $\mathrm{H}$ & -5.263304 & 0.760314 & 1.248598 \\
\hline $\mathrm{H}$ & -5.443102 & -0.817604 & 0.443787 \\
\hline $\mathrm{H}$ & -4.175489 & -0.427564 & 2.943702 \\
\hline $\mathrm{H}$ & -3.771163 & -2.399548 & -1.427034 \\
\hline $\mathrm{H}$ & 3.172043 & 0.909251 & 2.573794 \\
\hline $\mathrm{H}$ & 2.641263 & -0.772748 & 2.647336 \\
\hline $\mathrm{H}$ & 4.299302 & -0.346096 & 3.131035 \\
\hline $\mathrm{H}$ & 5.232641 & -1.251189 & -1.361804 \\
\hline $\mathrm{H}$ & 6.628468 & -0.314657 & -0.795344 \\
\hline $\mathrm{H}$ & 6.534922 & -2.031701 & -0.405104 \\
\hline C & -1.657535 & 1.428848 & -0.645802 \\
\hline $\mathrm{H}$ & -1.697579 & 0.806889 & -1.546309 \\
\hline C & -0.275834 & 1.272048 & 0.079706 \\
\hline $\mathrm{H}$ & -0.432786 & 0.895102 & 1.090953 \\
\hline C & -0.536108 & -2.003370 & -0.299808 \\
\hline C & 0.332986 & -2.879406 & 0.586309 \\
\hline $\mathrm{H}$ & 1.002588 & -2.271059 & 1.202301 \\
\hline $\mathrm{H}$ & 0.935655 & -3.541305 & -0.046850 \\
\hline $\mathrm{H}$ & -0.293550 & -3.497848 & 1.240053 \\
\hline \multicolumn{4}{|c|}{ orr } \\
\hline C & 1.143538 & 1.694116 & 1.210764 \\
\hline C & 1.069901 & 2.993709 & 0.492637 \\
\hline $\mathrm{O}$ & 0.007839 & 2.984225 & -0.347842 \\
\hline $\mathrm{O}$ & 1.789867 & 3.966125 & 0.592719 \\
\hline C & 2.047190 & 1.476215 & 2.172326 \\
\hline C & -3.151488 & 1.093592 & -0.107316 \\
\hline C & -2.773752 & -0.335978 & -0.178466 \\
\hline C & -2.164864 & 2.003272 & -0.142436 \\
\hline C & -0.119452 & -1.815842 & 0.913250 \\
\hline C & 0.447998 & -0.607382 & 0.144463 \\
\hline C & -4.611349 & 1.465272 & 0.034148 \\
\hline 0 & -5.378038 & 1.116632 & -1.127265 \\
\hline $\mathrm{O}$ & -2.332479 & -0.850128 & 0.994615 \\
\hline C & -2.656785 & -1.176950 & -1.243743 \\
\hline C & -1.949472 & -2.355889 & -0.782680 \\
\hline $\mathrm{O}$ & -1.592698 & -3.354860 & -1.401283 \\
\hline $\mathrm{O}$ & 1.884072 & -0.810738 & 0.171088 \\
\hline C & 2.603396 & -0.179727 & -0.786344 \\
\hline C & 4.066050 & -0.450960 & -0.759826 \\
\hline C & 4.889307 & 0.597744 & -1.475003 \\
\hline C & 4.646557 & -1.539789 & -0.209272 \\
\hline C & 4.058341 & -2.735280 & 0.476432 \\
\hline
\end{tabular}




\begin{tabular}{|c|c|c|c|}
\hline $\mathrm{O}$ & 2.080039 & 0.582785 & -1.587172 \\
\hline $\mathrm{H}$ & -2.418325 & 3.062978 & -0.078747 \\
\hline $\mathrm{H}$ & 0.145000 & -0.653259 & -0.905948 \\
\hline $\mathrm{H}$ & 5.736998 & -1.573738 & -0.295612 \\
\hline $\mathrm{H}$ & 2.095130 & 0.539978 & 2.724513 \\
\hline $\mathrm{H}$ & 2.768643 & 2.252448 & 2.424722 \\
\hline $\mathrm{H}$ & 0.071925 & -1.700548 & 1.988096 \\
\hline $\mathrm{H}$ & 0.416898 & -2.710505 & 0.574712 \\
\hline $\mathrm{H}$ & -5.040122 & 0.990422 & 0.929865 \\
\hline $\mathrm{H}$ & -4.694646 & 2.551915 & 0.145798 \\
\hline $\mathrm{H}$ & -5.592758 & 0.175512 & -1.092996 \\
\hline $\mathrm{H}$ & -2.949965 & -0.962162 & -2.265152 \\
\hline $\mathrm{H}$ & 4.624745 & 0.647445 & -2.540355 \\
\hline $\mathrm{H}$ & 4.710612 & 1.597820 & -1.055273 \\
\hline $\mathrm{H}$ & 5.957540 & 0.367944 & -1.391124 \\
\hline $\mathrm{H}$ & 4.244168 & -2.675969 & 1.561132 \\
\hline $\mathrm{H}$ & 2.983693 & -2.844796 & 0.320070 \\
\hline $\mathrm{H}$ & 4.569953 & -3.642316 & 0.124722 \\
\hline C & -0.692837 & 1.706360 & -0.342110 \\
\hline $\mathrm{H}$ & -0.538059 & 1.288219 & -1.344721 \\
\hline C & 0.030568 & 0.800312 & 0.718723 \\
\hline $\mathrm{H}$ & -0.654200 & 0.621134 & 1.550739 \\
\hline C & -1.621653 & -2.098856 & 0.704994 \\
\hline C & -2.143997 & -3.197596 & 1.617635 \\
\hline $\mathrm{H}$ & -1.942077 & -2.953892 & 2.667496 \\
\hline $\mathrm{H}$ & -1.643050 & -4.140761 & 1.368827 \\
\hline $\mathrm{H}$ & -3.224095 & -3.328272 & 1.481293 \\
\hline \multicolumn{4}{|c|}{50} \\
\hline C & 0.584586 & 2.538476 & 0.210713 \\
\hline C & -0.234655 & 3.498044 & -0.576307 \\
\hline $\mathrm{O}$ & -1.385161 & 2.888977 & -0.962254 \\
\hline 0 & -0.000849 & 4.651480 & -0.871417 \\
\hline C & 1.701066 & 2.922974 & 0.839590 \\
\hline C & -3.380533 & 0.288205 & 0.684802 \\
\hline C & -2.751059 & -0.970900 & 0.239374 \\
\hline C & -2.722922 & 1.426871 & 0.392374 \\
\hline C & 0.067452 & -1.149497 & -1.126952 \\
\hline C & 0.748471 & 0.129500 & -0.588148 \\
\hline C & -4.700849 & 0.264830 & 1.417948 \\
\hline $\mathrm{O}$ & -5.762205 & -0.240839 & 0.596999 \\
\hline $\mathrm{O}$ & -1.481597 & -1.088905 & 0.697111 \\
\hline C & -3.105419 & -1.935611 & -0.655429 \\
\hline C & -1.908831 & -2.711803 & -0.941162 \\
\hline $\mathrm{O}$ & -1.753999 & -3.664864 & -1.700027 \\
\hline $\mathrm{O}$ & 1.831262 & -0.204641 & 0.320291 \\
\hline C & 3.060669 & -0.446413 & -0.218885 \\
\hline C & 4.067628 & -0.729733 & 0.841006 \\
\hline C & 3.560623 & -1.045597 & 2.230930 \\
\hline
\end{tabular}




\begin{tabular}{|c|c|c|c|}
\hline C & 5.392429 & -0.696190 & 0.573118 \\
\hline C & 6.115332 & -0.374198 & -0.698525 \\
\hline 0 & 3.256369 & -0.429190 & -1.423249 \\
\hline $\mathrm{H}$ & -3.142915 & 2.382996 & 0.709247 \\
\hline $\mathrm{H}$ & 1.192796 & 0.612233 & -1.466082 \\
\hline $\mathrm{H}$ & 6.046744 & -0.927115 & 1.419249 \\
\hline $\mathrm{H}$ & 2.285066 & 2.239336 & 1.451523 \\
\hline $\mathrm{H}$ & 2.048032 & 3.951849 & 0.749773 \\
\hline $\mathrm{H}$ & 0.848667 & -1.772048 & -1.578316 \\
\hline $\mathrm{H}$ & -0.574770 & -0.836834 & -1.960366 \\
\hline $\mathrm{H}$ & -4.651990 & -0.414276 & 2.278380 \\
\hline $\mathrm{H}$ & -4.930171 & 1.275869 & 1.784379 \\
\hline $\mathrm{H}$ & -5.892258 & 0.371132 & -0.140294 \\
\hline $\mathrm{H}$ & -4.067937 & -2.040484 & -1.141954 \\
\hline $\mathrm{H}$ & 2.993272 & -0.206545 & 2.655442 \\
\hline $\mathrm{H}$ & 2.890231 & -1.916242 & 2.227060 \\
\hline $\mathrm{H}$ & 4.402123 & -1.265657 & 2.897858 \\
\hline $\mathrm{H}$ & 6.465675 & -1.303940 & -1.176849 \\
\hline $\mathrm{H}$ & 5.493123 & 0.158865 & -1.421113 \\
\hline $\mathrm{H}$ & 7.013844 & 0.216392 & -0.470839 \\
\hline C & -1.478792 & 1.517596 & -0.473007 \\
\hline $\mathrm{H}$ & -1.633866 & 0.912350 & -1.372655 \\
\hline C & -0.095613 & 1.187573 & 0.188165 \\
\hline $\mathrm{H}$ & -0.249944 & 0.821265 & 1.203526 \\
\hline C & -0.763908 & -2.028235 & -0.153146 \\
\hline C & 0.034457 & -2.992675 & 0.706159 \\
\hline $\mathrm{H}$ & 0.802514 & -2.462163 & 1.277481 \\
\hline $\mathrm{H}$ & 0.519618 & -3.731878 & 0.057796 \\
\hline $\mathrm{H}$ & -0.629208 & -3.520549 & 1.401405 \\
\hline & \multicolumn{3}{|c|}{----- Conformation 14 --------------- } \\
\hline C & 0.370772 & 2.508034 & 0.102374 \\
\hline C & -0.497334 & 3.377178 & -0.736114 \\
\hline $\mathrm{O}$ & -1.586120 & 2.670663 & -1.131075 \\
\hline $\mathrm{O}$ & -0.342832 & 4.535965 & -1.062501 \\
\hline C & 1.437003 & 2.995726 & 0.746756 \\
\hline C & -3.419350 & -0.025054 & 0.543161 \\
\hline C & -2.682901 & -1.240449 & 0.148242 \\
\hline C & -2.845142 & 1.153287 & 0.240523 \\
\hline C & 0.178027 & -1.241377 & -1.142249 \\
\hline C & 0.742076 & 0.098457 & -0.616351 \\
\hline C & -4.751830 & -0.182692 & 1.243969 \\
\hline $\mathrm{O}$ & -5.421653 & 1.042621 & 1.497081 \\
\hline $\mathrm{O}$ & -1.422249 & -1.257311 & 0.640365 \\
\hline C & -2.944602 & -2.246376 & -0.733475 \\
\hline C & -1.687242 & -2.938007 & -0.974607 \\
\hline 0 & -1.444617 & -3.890259 & -1.710480 \\
\hline $\mathrm{O}$ & 1.816542 & -0.130517 & 0.334125 \\
\hline C & 3.078841 & -0.285278 & -0.158654 \\
\hline
\end{tabular}




\begin{tabular}{|c|c|c|c|}
\hline C & 4.068477 & -0.462712 & 0.939951 \\
\hline C & 3.541554 & -0.796433 & 2.318246 \\
\hline C & 5.394156 & -0.319911 & 0.716240 \\
\hline C & 6.131739 & 0.040398 & -0.536523 \\
\hline $\mathrm{O}$ & 3.313780 & -0.279651 & -1.356115 \\
\hline $\mathrm{H}$ & -3.347996 & 2.079100 & 0.520029 \\
\hline $\mathrm{H}$ & 1.177853 & 0.591576 & -1.492885 \\
\hline $\mathrm{H}$ & 6.035434 & -0.477895 & 1.588727 \\
\hline $\mathrm{H}$ & 2.054197 & 2.377110 & 1.394396 \\
\hline $\mathrm{H}$ & 1.707281 & 4.045096 & 0.633497 \\
\hline $\mathrm{H}$ & 1.018998 & -1.813222 & -1.551459 \\
\hline $\mathrm{H}$ & -0.456654 & -0.998629 & -2.004129 \\
\hline $\mathrm{H}$ & -5.383400 & -0.869824 & 0.656425 \\
\hline $\mathrm{H}$ & -4.595623 & -0.655454 & 2.224044 \\
\hline $\mathrm{H}$ & -5.730307 & 1.409289 & 0.657511 \\
\hline $\mathrm{H}$ & -3.881994 & -2.432098 & -1.245842 \\
\hline $\mathrm{H}$ & 2.891905 & -0.000936 & 2.707119 \\
\hline $\mathrm{H}$ & 2.946817 & -1.720412 & 2.309171 \\
\hline $\mathrm{H}$ & 4.374884 & -0.933906 & 3.016831 \\
\hline $\mathrm{H}$ & 6.585104 & -0.862761 & -0.977740 \\
\hline $\mathrm{H}$ & 5.490868 & 0.496940 & -1.294320 \\
\hline $\mathrm{H}$ & 6.962339 & 0.717377 & -0.291779 \\
\hline C & -1.590276 & 1.310852 & -0.598733 \\
\hline $\mathrm{H}$ & -1.673608 & 0.669974 & -1.482883 \\
\hline C & -0.203534 & 1.108877 & 0.104723 \\
\hline $\mathrm{H}$ & -0.356829 & 0.761145 & 1.126759 \\
\hline C & -0.616111 & -2.158174 & -0.171965 \\
\hline C & 0.225307 & -3.045889 & 0.728160 \\
\hline $\mathrm{H}$ & 0.936144 & -2.449997 & 1.309028 \\
\hline $\mathrm{H}$ & 0.781398 & -3.759060 & 0.108254 \\
\hline $\mathrm{H}$ & -0.417409 & -3.608242 & 1.416003 \\
\hline & \multicolumn{3}{|c|}{--- Conformation 15 --- } \\
\hline C & 0.297022 & 2.694878 & 0.117706 \\
\hline C & -0.639866 & 3.524278 & -0.685942 \\
\hline $\mathrm{O}$ & -1.713817 & 2.770954 & -1.035137 \\
\hline $\mathrm{O}$ & -0.548244 & 4.687197 & -1.019567 \\
\hline C & 1.367140 & 3.230652 & 0.715144 \\
\hline C & -3.362345 & -0.001572 & 0.707510 \\
\hline C & -2.596322 & -1.185494 & 0.268760 \\
\hline C & -2.847941 & 1.200095 & 0.382700 \\
\hline C & 0.212000 & -1.055202 & -1.126567 \\
\hline C & 0.745633 & 0.295966 & -0.599545 \\
\hline C & -4.654717 & -0.166979 & 1.471293 \\
\hline $\mathrm{O}$ & -5.664593 & -0.814412 & 0.685853 \\
\hline $\mathrm{O}$ & -1.316834 & -1.151641 & 0.713097 \\
\hline C & -2.846806 & -2.193481 & -0.613145 \\
\hline C & -1.572157 & -2.830282 & -0.905613 \\
\hline $\mathrm{O}$ & -1.316799 & -3.766933 & -1.657712 \\
\hline
\end{tabular}




\begin{tabular}{|c|c|c|c|}
\hline 0 & 1.838591 & 0.077242 & 0.331829 \\
\hline C & 3.092336 & -0.075507 & -0.183861 \\
\hline C & 4.088363 & -0.335643 & 0.891766 \\
\hline C & 3.671017 & -0.069880 & 2.321457 \\
\hline C & 5.322125 & -0.807505 & 0.602742 \\
\hline C & 5.934115 & -1.177967 & -0.712962 \\
\hline 0 & 3.316902 & 0.007346 & -1.380474 \\
\hline $\mathrm{H}$ & -3.368924 & 2.107682 & 0.692397 \\
\hline $\mathrm{H}$ & 1.155833 & 0.810160 & -1.476027 \\
\hline $\mathrm{H}$ & 5.979066 & -0.958015 & 1.464841 \\
\hline $\mathrm{H}$ & 2.035963 & 2.642492 & 1.339025 \\
\hline $\mathrm{H}$ & 1.587538 & 4.289915 & 0.587400 \\
\hline $\mathrm{H}$ & 1.057376 & -1.588745 & -1.576566 \\
\hline $\mathrm{H}$ & -0.465150 & -0.827434 & -1.959469 \\
\hline $\mathrm{H}$ & -4.506186 & -0.816084 & 2.343475 \\
\hline $\mathrm{H}$ & -4.996254 & 0.816995 & 1.823593 \\
\hline $\mathrm{H}$ & -5.882689 & -0.239087 & -0.060035 \\
\hline $\mathrm{H}$ & -3.795797 & -2.412671 & -1.088038 \\
\hline $\mathrm{H}$ & 3.361759 & 0.974865 & 2.465231 \\
\hline $\mathrm{H}$ & 2.820721 & -0.698612 & 2.617827 \\
\hline $\mathrm{H}$ & 4.505376 & -0.274517 & 3.002065 \\
\hline $\mathrm{H}$ & 6.541430 & -2.086144 & -0.592503 \\
\hline $\mathrm{H}$ & 5.193999 & -1.326888 & -1.502464 \\
\hline $\mathrm{H}$ & 6.623582 & -0.383179 & -1.042852 \\
\hline C & -1.635766 & 1.413974 & -0.505475 \\
\hline $\mathrm{H}$ & -1.727212 & 0.767535 & -1.384603 \\
\hline C & -0.216471 & 1.272245 & 0.147175 \\
\hline $\mathrm{H}$ & -0.319200 & 0.924260 & 1.175714 \\
\hline C & -0.505246 & -2.012822 & -0.136214 \\
\hline C & 0.405486 & -2.873051 & 0.723237 \\
\hline $\mathrm{H}$ & 1.111903 & -2.255119 & 1.286417 \\
\hline $\mathrm{H}$ & 0.968059 & -3.555423 & 0.074995 \\
\hline $\mathrm{H}$ & -0.188450 & -3.470240 & 1.425465 \\
\hline-- & \multicolumn{3}{|c|}{---- Conformation 16 -------------- } \\
\hline C & 0.041885 & 2.644618 & 0.015536 \\
\hline C & -0.949470 & 3.367764 & -0.825108 \\
\hline 0 & -1.938387 & 2.511634 & -1.183672 \\
\hline 0 & -0.958094 & 4.528703 & -1.179129 \\
\hline C & 1.045273 & 3.289077 & 0.621436 \\
\hline C & -3.365894 & -0.369708 & 0.571106 \\
\hline C & -2.479752 & -1.482729 & 0.181637 \\
\hline C & -2.959144 & 0.868673 & 0.238697 \\
\hline C & 0.336986 & -1.119851 & -1.148792 \\
\hline C & 0.729875 & 0.282766 & -0.632425 \\
\hline C & -4.652542 & -0.690850 & 1.301165 \\
\hline 0 & -5.478743 & 0.437756 & 1.542124 \\
\hline $\mathrm{O}$ & -1.221901 & -1.326530 & 0.656399 \\
\hline C & -2.617499 & -2.525223 & -0.685474 \\
\hline
\end{tabular}




$\begin{array}{lrrr}\mathrm{C} & -1.283057 & -3.047472 & -0.937407 \\ \mathrm{O} & -0.925941 & -3.968106 & -1.666944 \\ \mathrm{O} & 1.805612 & 0.178133 & 0.338423 \\ \mathrm{C} & 3.084947 & 0.127190 & -0.131515 \\ \mathrm{C} & 4.061042 & -0.022404 & 0.982800 \\ \mathrm{C} & 3.569159 & 0.225685 & 2.391873 \\ \mathrm{C} & 5.342976 & -0.381292 & 0.745784 \\ \mathrm{C} & 6.033926 & -0.713051 & -0.540916 \\ \mathrm{O} & 3.343761 & 0.206578 & -1.321474 \\ \mathrm{H} & -3.576199 & 1.724443 & 0.512538 \\ \mathrm{H} & 1.122973 & 0.815758 & -1.505578 \\ \mathrm{H} & 5.979307 & -0.455866 & 1.632878 \\ \mathrm{H} & 1.752899 & 2.778619 & 1.270463 \\ \mathrm{H} & 1.169386 & 4.361437 & 0.474471 \\ \mathrm{H} & 1.239456 & -1.580241 & -1.567620 \\ \mathrm{H} & -0.336595 & -0.969160 & -2.001836 \\ \mathrm{H} & -5.194463 & -1.470889 & 0.740776 \\ \mathrm{H} & -4.416864 & -1.115228 & 2.287551 \\ \mathrm{H} & -5.850674 & 0.737652 & 0.701675 \\ \mathrm{H} & -3.528645 & -2.838644 & -1.182808 \\ \mathrm{H} & 3.154694 & 1.237304 & 2.503956 \\ \mathrm{H} & 2.773762 & -0.477988 & 2.672050 \\ \mathrm{H} & 4.394642 & 0.114912 & 3.104308 \\ \mathrm{H} & 6.725082 & -1.552465 & -0.380643 \\ \mathrm{H} & 5.339748 & -0.951382 & -1.350078 \\ \mathrm{H} & 6.650388 & 0.141140 & -0.866851 \\ \mathrm{C} & -1.748712 & 1.176447 & -0.623806 \\ \mathrm{H} & -1.754162 & 0.511120 & -1.493418 \\ \mathrm{C} & -0.339452 & 1.181456 & 0.064634 \\ \mathrm{H} & -0.437627 & 0.847252 & 1.098270 \\ \mathrm{C} & -0.314380 & -2.123429 & -0.158422 \\ \mathrm{C} & 0.649724 & -2.882533 & 0.737266 \\ \mathrm{H} & 1.282519 & -2.192956 & 1.304747 \\ \mathrm{H} & 1.288234 & -3.520233 & 0.114384 \\ \mathrm{H} & 0.096316 & -3.520751 & 1.436520\end{array}$


Table S5: Experimental and Boltzmann population weighted $[\alpha]$ values of $(-)-\left(6 R, 7 R, 8 S, 10 R, 2^{\prime} Z\right)-$ centratherin

\begin{tabular}{cccc}
\hline $\begin{array}{c}\text { Wavelength } \\
(\mathbf{n m})\end{array}$ & $\begin{array}{c}\text { CAM-B3LYP/Aug-cc- } \\
\text { pVDZ/PCM }\end{array}$ & $\begin{array}{c}{[\alpha]} \\
\text { Experiment/Acetonitrile }\end{array}$ & $\begin{array}{c}\text { Error in } \\
\text { Experimental } \\
{[\alpha]}\end{array}$ \\
\hline 365 & 74.8983 & 1224.2754 & 122.5047 \\
405 & -25.3814 & 300.7246 & 30.2899 \\
436 & -45.9587 & 132.2464 & 13.5386 \\
546 & -48.2192 & -0.7246 & -2.8995 \\
589 & -43.7214 & -12.3188 & -3.1495 \\
633 & -39.1880 & -17.0290 & -3.3618 \\
\hline
\end{tabular}


Table S6: Vibrational frequencies $\left(\mathrm{cm}^{-1}\right)$, rotational $\left(10^{-44} \mathrm{esu}^{2} \mathrm{~cm}^{2}\right)$ and dipole strengths $\left(10^{-40} \mathrm{esu}^{2} \mathrm{~cm}^{2}\right)$ for the four lowest energy conformers of $(-)-\left(6 R, 7 R, 8 S, 10 R, 2^{\prime} Z\right)$-centratherin.

\begin{tabular}{|c|c|c|c|c|c|}
\hline \multicolumn{3}{|c|}{ Conformer 1} & \multicolumn{3}{|c|}{ Conformer 2} \\
\hline position & Dip Str & $\begin{array}{l}\text { Rot. } \\
\text { Str. }\end{array}$ & position & Dip Str & $\begin{array}{l}\text { Rot. } \\
\text { Str. }\end{array}$ \\
\hline 16.94 & 875.79 & 0.13 & 17.86 & 565.65 & -0.37 \\
\hline 22.19 & 22.43 & -2.93 & 20.43 & 17.21 & -0.64 \\
\hline 38.80 & 138.34 & 1.09 & 37.98 & 176.29 & 8.29 \\
\hline 53.68 & 221.03 & -1.34 & 53.11 & 597.79 & -3.83 \\
\hline 61.10 & 355.89 & 7.78 & 55.11 & 145.43 & -1.82 \\
\hline 63.86 & 115.69 & -9.65 & 66.25 & 128.44 & -11.11 \\
\hline 81.16 & 172.23 & 8.54 & 77.53 & 48.94 & -0.49 \\
\hline 85.96 & 60.34 & 1.66 & 86.31 & 124.62 & 1.88 \\
\hline 91.97 & 109.85 & -5.63 & 96.10 & 190.97 & -19.25 \\
\hline 109.77 & 105.97 & -15.14 & 113.58 & 175.65 & 32.95 \\
\hline 116.92 & 172.89 & 12.88 & 118.27 & 86.26 & 2.21 \\
\hline 137.20 & 210.19 & -7.98 & 127.55 & 227.73 & -1.51 \\
\hline 170.17 & 125.50 & -28.75 & 162.38 & 118.70 & 34.66 \\
\hline 176.40 & 102.80 & 2.98 & 173.60 & 155.09 & -41.71 \\
\hline 192.22 & 11.51 & -2.79 & 193.98 & 9.23 & 3.59 \\
\hline 197.00 & 119.18 & -8.59 & 195.72 & 97.81 & 2.21 \\
\hline 203.95 & 19.63 & 2.09 & 201.37 & 35.04 & -5.72 \\
\hline 211.53 & 27.31 & 18.04 & 206.46 & 28.07 & -5.75 \\
\hline 220.84 & 34.71 & 2.91 & 219.29 & 32.14 & -11.51 \\
\hline 242.02 & 7.16 & 4.73 & 243.89 & 63.18 & 18.35 \\
\hline 249.80 & 23.71 & -0.15 & 248.34 & 64.21 & -9.86 \\
\hline 259.77 & 94.73 & -17.37 & 256.77 & 107.89 & -8.03 \\
\hline 271.72 & 138.86 & 24.49 & 264.84 & 118.70 & 51.40 \\
\hline 283.09 & 69.48 & -38.00 & 283.85 & 135.67 & -40.60 \\
\hline 293.45 & 139.06 & 27.14 & 299.72 & 175.47 & 52.52 \\
\hline 301.01 & 256.05 & 34.05 & 316.37 & 77.43 & -30.91 \\
\hline 323.09 & 44.53 & -30.26 & 323.49 & 30.42 & -8.61 \\
\hline 327.67 & 86.36 & -21.96 & 324.71 & 19.09 & -16.81 \\
\hline 347.39 & 1839.72 & 19.69 & 346.18 & 1858.32 & 23.88 \\
\hline 387.70 & 79.69 & -23.35 & 384.66 & 91.69 & 17.52 \\
\hline 395.59 & 37.92 & -6.97 & 393.86 & 3.80 & -4.88 \\
\hline 417.14 & 217.01 & 72.12 & 413.33 & 255.91 & 10.90 \\
\hline 439.15 & 81.15 & 3.07 & 439.63 & 195.24 & 9.27 \\
\hline 449.36 & 126.73 & 2.15 & 458.60 & 119.06 & -20.15 \\
\hline 478.03 & 27.46 & -4.98 & 475.47 & 48.54 & -2.07 \\
\hline 489.35 & 49.37 & -1.82 & 485.35 & 65.38 & -40.98 \\
\hline 511.70 & 35.01 & 7.47 & 524.53 & 120.01 & 3.40 \\
\hline
\end{tabular}




\begin{tabular}{|c|c|c|c|c|c|}
\hline 543.34 & 275.51 & -19.52 & 531.86 & 99.80 & 18.70 \\
\hline 574.60 & 49.12 & -0.51 & 573.47 & 8.77 & 10.68 \\
\hline 599.59 & 195.36 & 38.57 & 592.91 & 108.72 & -23.28 \\
\hline 603.74 & 30.86 & -29.11 & 600.68 & 127.97 & 24.12 \\
\hline 605.46 & 15.32 & 3.93 & 608.89 & 63.35 & -16.38 \\
\hline 624.60 & 26.56 & -18.03 & 618.26 & 62.16 & -32.82 \\
\hline 637.26 & 14.48 & 14.89 & 636.02 & 7.34 & 9.71 \\
\hline 649.47 & 19.50 & 1.21 & 669.22 & 37.47 & 2.24 \\
\hline 693.56 & 94.36 & -16.50 & 696.66 & 65.22 & -28.6 \\
\hline 717.90 & 39.11 & 27.05 & 717.27 & 28.39 & 21.43 \\
\hline 728.06 & 30.04 & -37.58 & 731.01 & 49.39 & -25.74 \\
\hline 746.78 & 85.45 & 48.15 & 748.46 & 76.27 & 37.30 \\
\hline 764.67 & 85.49 & -12.15 & 763.56 & 50.67 & -45.90 \\
\hline 771.49 & 143.18 & 52.00 & 764.72 & 95.86 & 0.4 \\
\hline 804.85 & 59.81 & 17.55 & 815.90 & 75.69 & -54.91 \\
\hline 821.01 & 207.30 & -28.94 & 821.91 & 144.95 & 20.12 \\
\hline 826.19 & 124.48 & 37.09 & 30.25 & 107.06 & 62.2 \\
\hline 841.76 & 83.72 & -42.86 & 838.80 & 118.39 & -49.4 \\
\hline 856.43 & 77.95 & 41.43 & 852.59 & 76.16 & 49.2 \\
\hline 867.15 & 31.22 & -6.96 & 866.29 & 27.02 & 24.3 \\
\hline 869.74 & 171.42 & -93.51 & 869.27 & 175.32 & -116.1 \\
\hline 891.09 & 142.51 & -24.96 & 896.84 & 130.50 & -35.6 \\
\hline 927.30 & 207.25 & 122.68 & 925.16 & 167.72 & 85.2 \\
\hline 932.99 & 159.44 & -54.03 & 934.08 & 88.98 & -50.13 \\
\hline 948.89 & 10.15 & 6.42 & 953.93 & 176.81 & 90.2 \\
\hline 960.81 & 168.17 & -30.55 & 960.90 & 150.13 & -54.23 \\
\hline 967.83 & 29.64 & 17.66 & 968.66 & 36.34 & 9.5 \\
\hline 977.97 & 351.89 & 0.23 & 976.24 & 386.10 & -63.27 \\
\hline 987.26 & 117.67 & -35.62 & 987.63 & 123.71 & -3.41 \\
\hline 1019.72 & 543.61 & 49.20 & 1018.42 & 661.15 & 101.31 \\
\hline 1027.27 & 443.25 & -113.50 & 1027.44 & 144.35 & 35.8 \\
\hline 1029.51 & 180.19 & 44.70 & 1029.36 & 470.19 & -112.58 \\
\hline 1041.85 & 401.34 & -142.76 & 1041.48 & 336.23 & -21.5 \\
\hline 1044.37 & 159.41 & 179.74 & 1044.09 & 160.97 & -9.1 \\
\hline 1052.64 & 421.02 & 134.58 & 1052.68 & 472.18 & 93.5 \\
\hline 1069.15 & 40.33 & -40.45 & 1068.90 & 40.08 & -40.15 \\
\hline 1079.06 & 135.72 & 20.18 & 1076.11 & 94.91 & -40.4 \\
\hline 1085.92 & 68.55 & 63.02 & 1085.12 & 55.08 & 36.77 \\
\hline 1104.35 & 175.08 & -78.52 & 1103.47 & 170.80 & -147.7 \\
\hline 1106.63 & 115.49 & -44.60 & 1105.16 & 47.67 & 67.0 \\
\hline 1112.02 & 321.87 & 291.20 & 1108.81 & 341.04 & 280.2 \\
\hline 1148.59 & 1213.91 & 171.75 & 1147.79 & 1301.11 & 377.9 \\
\hline 1154.33 & 827.12 & -367.08 & 1152.80 & 660.89 & -638.6 \\
\hline
\end{tabular}




\begin{tabular}{|c|c|c|c|c|c|}
\hline 1167.57 & 1248.31 & -157.83 & 1167.20 & 1275.63 & -60.10 \\
\hline 183.65 & 47.41 & 73.38 & 1184.11 & 47.88 & 98.28 \\
\hline 1191.96 & 125.81 & -136.24 & 1189.87 & 200.75 & -181.39 \\
\hline 1196.71 & 68.65 & 26.80 & 1197.52 & 66.19 & 69.79 \\
\hline 1215.36 & 86.33 & 74.54 & 1213.51 & 81.94 & 75.38 \\
\hline 1241.31 & 321.83 & -134.03 & 1242.47 & 396.50 & -97.87 \\
\hline 1248.27 & 718.09 & 123.69 & 1248.44 & 621.37 & 96.79 \\
\hline 1280.51 & 299.51 & 6.57 & 1280.33 & 198.08 & -10.22 \\
\hline 1290.57 & 462.83 & 116.91 & 1287.95 & 591.17 & 133.46 \\
\hline 1294.36 & 124.81 & 24.09 & 1294.51 & 79.60 & 5.41 \\
\hline 1313.60 & 558.18 & -161.58 & 1310.33 & 671.87 & -102.79 \\
\hline 1327.30 & 83.72 & 121.95 & 1326.67 & 73.76 & 155.40 \\
\hline 1348.61 & 55.29 & 72.66 & 1346.02 & 9.30 & 27.44 \\
\hline 1350.21 & 100.63 & 90.72 & 1347.98 & 99.82 & 56.88 \\
\hline 1360.03 & 37.33 & -8.12 & 1358.23 & 48.09 & -28.92 \\
\hline 1372.38 & 103.46 & -14.91 & 1372.43 & 110.06 & -18.89 \\
\hline 1379.15 & 84.91 & -50.00 & 1379.95 & 81.92 & -41.73 \\
\hline 1385.41 & 38.82 & -4.90 & 1385.35 & 29.55 & -2.67 \\
\hline 1389.72 & 115.90 & -21.97 & 1389.45 & 154.29 & -23.71 \\
\hline 1394.72 & 105.35 & -84.41 & 1394.92 & 215.43 & 45.89 \\
\hline 1396.74 & 191.35 & 58.89 & 1395.29 & 62.78 & 50.26 \\
\hline 1397.53 & 109.01 & 18.24 & 1397.15 & 57.98 & -7.73 \\
\hline 1401.36 & 53.61 & 5.68 & 1400.48 & 58.99 & 1.17 \\
\hline 1428.50 & 126.15 & -12.48 & 1429.95 & 120.82 & -11.56 \\
\hline 1439.25 & 51.41 & -30.03 & 1439.67 & 46.76 & -31.16 \\
\hline 1444.91 & 46.73 & -6.61 & 1444.86 & 49.92 & -5.23 \\
\hline 1449.14 & 28.36 & 4.31 & 1448.81 & 28.11 & 4.41 \\
\hline 1457.37 & 14.95 & 22.21 & 1456.25 & 12.00 & 19.40 \\
\hline 1465.14 & 77.18 & -4.86 & 1464.78 & 80.72 & -2.15 \\
\hline 1469.94 & 129.13 & 1.24 & 1469.63 & 128.37 & 1.12 \\
\hline 1471.00 & 19.01 & 4.09 & 1471.44 & 17.73 & 6.42 \\
\hline 1472.23 & 22.91 & -0.40 & 1474.37 & 1.48 & 1.40 \\
\hline 1602.18 & 1376.12 & 159.93 & 1607.35 & 1355.82 & 99.13 \\
\hline 1680.27 & 488.27 & 33.84 & 1680.58 & 482.91 & 37.14 \\
\hline 1701.60 & 650.36 & -87.39 & 1703.49 & 598.60 & -47.64 \\
\hline 1711.27 & 360.30 & 107.60 & 1711.80 & 354.16 & 123.75 \\
\hline 1717.14 & 903.82 & 424.30 & 1718.24 & 914.14 & 502.10 \\
\hline 1721.76 & 448.61 & -451.60 & 1721.90 & 444.90 & -566.62 \\
\hline 1766.74 & 1480.05 & -50.68 & 1766.85 & 1481.34 & -34.83 \\
\hline 3018.77 & 32.08 & 0.22 & 3018.88 & 32.54 & 0.15 \\
\hline 3033.25 & 47.22 & 1.38 & 3032.99 & 47.08 & 2.12 \\
\hline 3040.25 & 75.07 & -17.10 & 3040.73 & 75.05 & 23.63 \\
\hline 3046.83 & 19.79 & 2.21 & 3047.01 & 21.37 & 0.44 \\
\hline
\end{tabular}




\begin{tabular}{|c|c|c|c|c|c|}
\hline 3057.72 & 33.20 & -3.79 & 3058.44 & 32.99 & -4.92 \\
\hline 3066.57 & 12.27 & -5.69 & 3071.20 & 12.95 & -5.09 \\
\hline 3073.00 & 36.84 & -4.30 & 3073.07 & 37.29 & -4.30 \\
\hline 3090.56 & 24.81 & 0.24 & 3090.42 & 29.73 & -0.84 \\
\hline 3090.81 & 26.42 & 16.41 & 3093.04 & 20.25 & 17.37 \\
\hline 3096.26 & 30.22 & 6.73 & 3100.08 & 31.86 & -7.04 \\
\hline 3112.96 & 12.23 & -1.21 & 3113.52 & 11.76 & -0.09 \\
\hline 3119.80 & 13.19 & -0.61 & 3118.63 & 13.94 & -0.09 \\
\hline 3122.70 & 31.88 & -1.49 & 3122.24 & 30.29 & -1.33 \\
\hline 3129.20 & 32.76 & 0.20 & 3129.30 & 32.05 & -0.21 \\
\hline 3132.94 & 18.25 & 0.01 & 3132.83 & 19.23 & 0.12 \\
\hline 3137.86 & 29.50 & 3.24 & 3137.63 & 30.13 & 3.39 \\
\hline 3158.27 & 5.01 & -2.37 & 3158.29 & 4.93 & -2.31 \\
\hline 3165.08 & 19.94 & 4.94 & 3164.35 & 20.99 & 4.99 \\
\hline 3170.36 & 7.62 & -1.15 & 3171.83 & 7.28 & 1.96 \\
\hline 3257.45 & 1.01 & -0.37 & 3257.47 & 1.04 & -0.34 \\
\hline 3258.66 & 1.38 & -0.45 & 3262.66 & 1.47 & -0.27 \\
\hline 3790.10 & 62.72 & 6.06 & 3788.54 & 62.33 & -3.58 \\
\hline \multicolumn{3}{|c|}{ Conformer 4} & \multicolumn{3}{|c|}{ Conformer 3} \\
\hline position & \multicolumn{2}{|r|}{$\begin{array}{l}\text { Rot. } \\
\text { Str. }\end{array}$} & position & Dip Str & $\begin{array}{l}\text { Rot. } \\
\text { Str. }\end{array}$ \\
\hline 17.65 & 680.15 & 2.23 & 16.90 & 906.98 & -1.54 \\
\hline 21.54 & 37.50 & 0.08 & 22.70 & 23.61 & 0.92 \\
\hline 39.25 & 159.48 & 1.36 & 39.72 & 121.42 & 1.35 \\
\hline 53.93 & 177.18 & -2.83 & 54.20 & 157.35 & -2.45 \\
\hline 61.24 & 77.29 & -7.01 & 61.44 & 105.05 & -8.83 \\
\hline 69.80 & 294.67 & -11.83 & 71.26 & 249.05 & 9.42 \\
\hline 80.76 & 304.06 & -9.35 & 81.24 & 221.85 & 11.07 \\
\hline 84.93 & 6.61 & -0.62 & 84.85 & 57.00 & -21.73 \\
\hline 96.44 & 146.01 & 0.68 & 96.97 & 268.73 & -12.64 \\
\hline 111.15 & 15.69 & 7.61 & 110.63 & 63.90 & -8.11 \\
\hline 126.72 & 398.57 & 23.03 & 127.05 & 326.72 & 20.90 \\
\hline 135.47 & 12.56 & 8.49 & 136.16 & 15.30 & -0.39 \\
\hline 168.14 & 172.00 & -34.73 & 164.97 & 207.07 & 11.53 \\
\hline 175.54 & 118.70 & -24.11 & 175.73 & 139.57 & -29.56 \\
\hline 180.87 & 12.79 & 18.21 & 179.16 & 51.54 & 5.31 \\
\hline 194.60 & 16.49 & 2.57 & 194.90 & 12.78 & 2.40 \\
\hline 199.72 & 7.37 & 3.72 & 198.99 & 17.39 & -2.64 \\
\hline 209.47 & 32.60 & 21.18 & 209.64 & 37.85 & -10.30 \\
\hline 215.20 & 26.68 & -7.80 & 214.26 & 15.39 & -0.90 \\
\hline 237.90 & 14.55 & 9.71 & 238.55 & 11.47 & 8.49 \\
\hline 248.04 & 26.00 & 1.42 & 248.98 & 19.47 & 1.44 \\
\hline 257.61 & 47.29 & -16.51 & 258.08 & 11.10 & -4.01 \\
\hline
\end{tabular}




\begin{tabular}{|c|c|c|c|c|c|}
\hline 271.32 & 591.15 & -61.88 & 270.06 & 27.81 & 1.61 \\
\hline 82.74 & 77.41 & 27.23 & 282.67 & 214.10 & -65.86 \\
\hline 295.58 & 75.44 & 3.57 & 296.67 & 218.38 & 71.2 \\
\hline 314.14 & 942.49 & 115.88 & 318.71 & 371.74 & -29.95 \\
\hline 324.90 & 397.94 & 57.11 & 325.92 & 268.21 & 31.0 \\
\hline 329.16 & 619.03 & -67.01 & 329.03 & 1144.43 & 111.7 \\
\hline 342.08 & 375.36 & -69.66 & 343.54 & 864.80 & -59.2 \\
\hline 386.44 & 108.43 & -14.27 & 386.38 & 86.18 & -29.9 \\
\hline 390.65 & 9.88 & -3.78 & 390.99 & 4.24 & -5.02 \\
\hline 408.20 & 118.54 & 1.40 & 408.72 & 89.27 & 2. \\
\hline 425.71 & 216.70 & 44.01 & 427.36 & 87.51 & 26.5 \\
\hline 449.43 & 63.18 & -0.59 & 450.02 & 52.91 & -4.26 \\
\hline 476.11 & 42.09 & 7.30 & 477.37 & 90.51 & 4.8 \\
\hline 491.96 & 113.36 & -1.53 & 492.13 & 109.65 & -8.2 \\
\hline 507.50 & 223.06 & -76.36 & 505.50 & 273.14 & -66.3 \\
\hline 530.22 & 20.53 & 14.18 & 531.43 & 29.62 & 19.00 \\
\hline 567.58 & 16.21 & 16.06 & 567.55 & 15.58 & 24.9 \\
\hline 597.46 & 72.92 & -37.44 & 596.59 & 45.37 & -34.92 \\
\hline 600.90 & 162.30 & 37.52 & 600.91 & 161.22 & 39.9 \\
\hline 613.45 & 61.75 & -18.33 & 613.44 & 70.47 & -17.93 \\
\hline 627.54 & 3.12 & -2.03 & 626.14 & 12.20 & -2.55 \\
\hline 638.24 & 23.40 & 15.07 & 638.21 & 23.52 & 18.2 \\
\hline 684.10 & 63.94 & 2.53 & 685.31 & 69.20 & 9.7 \\
\hline 699.45 & 17.37 & -6.91 & 698.66 & 13.98 & -9.50 \\
\hline 706.66 & 17.08 & -1.88 & 706.35 & 17.65 & -3.67 \\
\hline 742.14 & 47.11 & -23.79 & 741.75 & 33.76 & -22.22 \\
\hline 746.23 & 83.79 & 13.00 & 746.04 & 83.77 & 14.5 \\
\hline 753.24 & 27.20 & -10.07 & 753.53 & 26.53 & -13.44 \\
\hline 764.70 & 90.91 & -13.44 & 764.83 & 91.80 & -13.8 \\
\hline 803.37 & 20.13 & -6.63 & 805.62 & 34.92 & 5.7 \\
\hline 820.08 & 219.38 & -1.34 & 819.62 & 210.98 & 5.4 \\
\hline 824.33 & 124.06 & 47.97 & 824.78 & 122.80 & 87.40 \\
\hline 836.61 & 125.76 & -5.90 & 836.16 & 130.14 & 9.5 \\
\hline 846.80 & 20.09 & -7.19 & 846.56 & 27.43 & 2.7 \\
\hline 865.57 & 85.71 & 41.07 & 865.48 & 115.51 & 17.6 \\
\hline 869.31 & 145.00 & -113.91 & 869.80 & 139.80 & -111.67 \\
\hline 890.27 & 61.06 & -19.75 & 891.61 & 120.50 & -20.56 \\
\hline 925.94 & 224.12 & 100.56 & 926.08 & 214.97 & 108.19 \\
\hline 931.98 & 99.19 & -68.14 & 933.19 & 113.65 & -61.5 \\
\hline 955.76 & 71.63 & 30.11 & 952.58 & 29.66 & -3.1 \\
\hline 961.05 & 141.66 & -44.31 & 961.08 & 156.65 & -31.1 \\
\hline 968.29 & 29.18 & -3.07 & 968.43 & 38.65 & 2. \\
\hline 985.11 & 155.89 & -87.54 & 984.80 & 51.71 & 84. \\
\hline
\end{tabular}




\begin{tabular}{|c|c|c|c|c|c|}
\hline 987.24 & 239.48 & 74.96 & 987.62 & 242.79 & -36.13 \\
\hline 1015.90 & 542.65 & 79.36 & 1016.24 & 580.68 & 119.96 \\
\hline 1025.60 & 129.50 & 2.02 & 1025.61 & 131.23 & -2.24 \\
\hline 1040.69 & 115.66 & -15.88 & 1041.40 & 203.81 & -113.71 \\
\hline 1043.05 & 331.47 & 20.24 & 1043.47 & 227.13 & 96.80 \\
\hline 1049.31 & 576.35 & -251.40 & 1049.76 & 567.43 & -263.60 \\
\hline 1053.20 & 658.62 & 190.03 & 1053.25 & 680.68 & 166.40 \\
\hline 1069.32 & 45.02 & -46.75 & 1069.29 & 45.71 & -48.12 \\
\hline 1083.88 & 118.40 & 8.12 & 1084.31 & 100.20 & 5.84 \\
\hline 1086.33 & 156.32 & 263.84 & 1086.69 & 159.16 & 236.17 \\
\hline 1104.68 & 122.32 & -68.18 & 1104.67 & 124.59 & -57.98 \\
\hline 1107.48 & 253.61 & -109.40 & 1107.30 & 288.45 & -33.33 \\
\hline 1111.52 & 271.30 & 283.41 & 1110.54 & 216.39 & 188.48 \\
\hline 1148.13 & 1112.87 & 104.60 & 1148.00 & 1128.12 & 147.52 \\
\hline 1155.08 & 899.93 & -279.90 & 1155.02 & 865.45 & -334.50 \\
\hline 1167.49 & 1262.57 & -163.96 & 1167.53 & 1270.59 & -160.55 \\
\hline 1183.91 & 56.30 & 91.19 & 1183.90 & 47.65 & 81.98 \\
\hline 1186.22 & 74.10 & -98.41 & 1186.08 & 76.97 & -96.62 \\
\hline 1196.50 & 50.03 & -9.21 & 1196.35 & 73.86 & 12.07 \\
\hline 1211.71 & 89.31 & 39.53 & 1210.90 & 88.69 & -60.12 \\
\hline 1242.82 & 494.04 & -92.03 & 1242.96 & 497.42 & -84.77 \\
\hline 1249.09 & 521.13 & 93.39 & 1249.16 & 521.63 & 92.42 \\
\hline 1280.72 & 200.48 & -3.90 & 1280.72 & 201.06 & -7.20 \\
\hline 1288.49 & 217.53 & 98.33 & 1287.84 & 207.76 & 92.01 \\
\hline 1293.03 & 111.73 & -12.57 & 1292.83 & 123.74 & -16.45 \\
\hline 1301.99 & 875.30 & -45.32 & 1301.79 & 868.57 & -22.31 \\
\hline 1321.78 & 130.32 & 131.37 & 1321.19 & 142.38 & 129.45 \\
\hline 1347.03 & 18.45 & 73.72 & 1346.44 & 14.77 & 71.78 \\
\hline 1355.81 & 59.95 & -2.96 & 1355.35 & 50.29 & 8.86 \\
\hline 1362.02 & 331.19 & 28.12 & 1362.27 & 282.59 & 65.49 \\
\hline 1372.26 & 117.99 & -28.51 & 1372.37 & 117.55 & -27.94 \\
\hline 1379.06 & 18.22 & 3.24 & 1377.42 & 73.73 & -58.84 \\
\hline 1384.08 & 81.72 & -7.03 & 1382.69 & 96.45 & 53.86 \\
\hline 1385.62 & 58.98 & 6.51 & 1385.42 & 34.84 & -12.30 \\
\hline 1393.69 & 15.74 & -5.03 & 1393.64 & 16.84 & -0.11 \\
\hline 1397.08 & 23.82 & -3.01 & 1396.99 & 24.46 & -5.62 \\
\hline 1400.67 & 58.89 & 12.95 & 1400.66 & 59.43 & 7.66 \\
\hline 1403.54 & 141.11 & 15.52 & 1403.46 & 163.09 & -8.20 \\
\hline 1433.07 & 117.09 & -8.43 & 1432.83 & 113.90 & -8.07 \\
\hline 1439.48 & 48.94 & -30.81 & 1439.38 & 47.88 & -31.24 \\
\hline 1444.93 & 49.17 & -6.02 & 1444.92 & 49.87 & -5.51 \\
\hline 1448.67 & 28.22 & 4.22 & 1448.91 & 28.21 & 4.20 \\
\hline 1455.54 & 12.90 & 20.55 & 1455.92 & 11.85 & 19.95 \\
\hline
\end{tabular}




\begin{tabular}{|c|c|c|c|c|c|}
\hline 1463.69 & 80.64 & -5.80 & 1464.06 & 82.88 & -5.38 \\
\hline 1469.82 & 125.46 & 0.99 & 1469.94 & 125.26 & 0.63 \\
\hline 1471.27 & 18.73 & 1.14 & 1471.04 & 17.72 & -0.99 \\
\hline 1472.00 & 38.47 & 9.21 & 1471.53 & 40.29 & -0.16 \\
\hline 1604.66 & 1412.70 & 83.64 & 1604.21 & 1401.27 & 79.69 \\
\hline 1680.44 & 484.68 & 38.17 & 1680.46 & 485.99 & 39.30 \\
\hline 1708.44 & 883.31 & 0.46 & 1707.77 & 876.62 & -14.71 \\
\hline 1712.27 & 472.17 & 167.98 & 1712.31 & 439.69 & 153.21 \\
\hline 1720.97 & 603.42 & 567.96 & 1720.54 & 632.10 & 653.18 \\
\hline 1722.89 & 372.41 & -713.65 & 1722.42 & 385.75 & -780.92 \\
\hline 1765.16 & 1469.85 & -42.16 & 1765.23 & 1471.13 & -38.52 \\
\hline 3014.59 & 60.20 & 11.02 & 3012.59 & 60.60 & -14.91 \\
\hline 3018.52 & 32.22 & -0.02 & 3018.55 & 32.42 & -0.03 \\
\hline 3032.99 & 46.88 & 1.48 & 3033.11 & 46.92 & 1.58 \\
\hline 3046.94 & 20.23 & 2.46 & 3046.91 & 20.21 & 2.47 \\
\hline 3057.69 & 32.76 & -3.24 & 3057.77 & 31.89 & -3.24 \\
\hline 3067.86 & 16.17 & -2.38 & 3068.15 & 11.71 & -5.90 \\
\hline 3070.55 & 18.94 & -6.80 & 3071.03 & 24.43 & 6.23 \\
\hline 3072.39 & 36.95 & -4.09 & 3072.41 & 36.84 & -4.07 \\
\hline 3090.36 & 28.86 & -0.34 & 3090.43 & 26.25 & -0.04 \\
\hline 3091.47 & 21.37 & 17.98 & 3090.81 & 24.83 & 17.89 \\
\hline 3112.92 & 12.90 & -0.76 & 3112.97 & 13.70 & -0.90 \\
\hline 3119.68 & 12.22 & 1.26 & 3120.25 & 11.02 & 1.49 \\
\hline 3122.41 & 30.72 & -1.63 & 3122.63 & 31.06 & -1.52 \\
\hline 3129.28 & 31.68 & 0.02 & 3129.31 & 31.69 & -0.08 \\
\hline 3132.90 & 18.86 & -0.02 & 3132.89 & 18.85 & 0.19 \\
\hline 3137.61 & 30.74 & 3.29 & 3137.58 & 30.18 & 3.30 \\
\hline 3157.97 & 5.15 & -2.48 & 3157.85 & 5.15 & -2.53 \\
\hline 3164.98 & 19.94 & 5.03 & 3164.73 & 20.15 & 5.02 \\
\hline 3185.80 & 0.46 & -0.21 & 3185.63 & 0.55 & -0.33 \\
\hline 3257.16 & 1.13 & -0.39 & 3256.99 & 1.13 & -0.35 \\
\hline 3259.01 & 1.64 & -0.29 & 3259.06 & 1.59 & -0.33 \\
\hline 3794.59 & 76.49 & 13.14 & 3791.73 & 77.53 & -9.02 \\
\hline
\end{tabular}


Table S7: Electronic dipole strengths and Rotational Strengths $(R)$ of four lowest energy conformers of ()-(6R,7R,8S,10R,2'Z)-centratherin.

C1

\begin{tabular}{|c|c|c|}
\hline position (nm) & Dip. Str. & $\mathrm{R}$ (velocity) \\
\hline 296.42 & 0.0101 & 7.1431 \\
\hline 251.66 & 2.2303 & 7.8894 \\
\hline 244.58 & 0.0096 & 9.6569 \\
\hline 240.69 & 0.1152 & 6.3826 \\
\hline 221.78 & 3.0986 & -102.75 \\
\hline 216.59 & 0.1522 & 3.1489 \\
\hline 212.2 & 1.7331 & 72.5724 \\
\hline 206.29 & 0.1844 & 8.0921 \\
\hline 206.07 & 0.0787 & 10.3909 \\
\hline 199.7 & 0.0166 & 7.7012 \\
\hline 194.17 & 0.1621 & 6.7335 \\
\hline 191.84 & 0.1204 & 2.0987 \\
\hline 187.08 & 0.7808 & -39.9301 \\
\hline 185.41 & 0.5367 & 3.0376 \\
\hline 185.28 & 0.3207 & 12.5472 \\
\hline 184.89 & 0.3389 & -17.8179 \\
\hline 183.51 & 0.1022 & 15.4826 \\
\hline 182.96 & 0.1011 & 0.7986 \\
\hline 182.7 & 0.1723 & -6.8649 \\
\hline 181.39 & 0.0329 & -8.1229 \\
\hline 180.7 & 0.0809 & -14.2656 \\
\hline
\end{tabular}




\begin{tabular}{|c|c|c|}
\hline 179.4 & 0.1545 & -52.0477 \\
\hline 177.67 & 0.0585 & 29.7352 \\
\hline 176.47 & 0.3223 & 38.732 \\
\hline 175.77 & 0.086 & 14.3868 \\
\hline 175.08 & 0.1328 & 8.4614 \\
\hline 174.6 & 0.1595 & 0.1597 \\
\hline 173.74 & 0.1091 & -38.0596 \\
\hline 173.34 & 0.0258 & -11.3297 \\
\hline 172.21 & 0.0325 & 10.282 \\
\hline 170.78 & 0.1733 & 13.5633 \\
\hline 170.24 & 0.0934 & -14.3695 \\
\hline 170.15 & 0.1418 & -6.0529 \\
\hline 169.59 & 0.2399 & -1.2614 \\
\hline 169.29 & 0.076 & -41.3766 \\
\hline 169.17 & 0.07 & -11.5681 \\
\hline 168.29 & 0.0252 & 13.0897 \\
\hline 167.49 & 0.0271 & 22.853 \\
\hline 166.95 & 0.0785 & -12.3698 \\
\hline 165.53 & 0.0052 & -13.7804 \\
\hline
\end{tabular}

C2

\begin{tabular}{|l|l|l|}
\hline position $(\mathrm{nm})$ & Dip. Str. & R (velocity) \\
\hline $\mathbf{2 9 6 . 5 9}$ & 0.0194 & 15.518 \\
\hline $\mathbf{2 4 7 . 4 4}$ & 2.0346 & -11.5861 \\
\hline $\mathbf{2 4 4 . 5 2}$ & 0.0302 & 18.9863 \\
\hline
\end{tabular}




\begin{tabular}{|c|c|c|}
\hline 240.42 & 0.1232 & 5.6968 \\
\hline 221.66 & 3.0512 & -91.3068 \\
\hline 217.32 & 0.0582 & -2.7733 \\
\hline 212.01 & 1.7777 & 67.1247 \\
\hline 206.93 & 0.0999 & 2.9098 \\
\hline 206.47 & 0.0347 & 16.8313 \\
\hline 197.29 & 0.0665 & 12.1478 \\
\hline 195.11 & 0.2537 & 6.0235 \\
\hline 190.39 & 1.6434 & -32.9539 \\
\hline 187.75 & 0.0546 & -22.1055 \\
\hline 187.13 & 0.2047 & 6.6942 \\
\hline 185.26 & 0.0093 & -3.3543 \\
\hline 184.4 & 0.625 & 79.4465 \\
\hline 183.65 & 0.0893 & 4.9692 \\
\hline 183.37 & 0.0788 & -1.9912 \\
\hline 182.89 & 0.1085 & -40.9582 \\
\hline 181.11 & 0.1768 & -21.5977 \\
\hline 179.38 & 0.0884 & 2.8871 \\
\hline 179.18 & 0.0736 & 3.3342 \\
\hline 178.84 & 0.2201 & -62.0584 \\
\hline 176.72 & 0.0691 & 13.5688 \\
\hline 174.74 & 0.0825 & 21.2481 \\
\hline 174.37 & 0.0699 & -29.5493 \\
\hline 173.58 & 0.1343 & 50.1979 \\
\hline 173.07 & 0.0292 & -29.197 \\
\hline
\end{tabular}




\begin{tabular}{|l|l|l|}
\hline 172.16 & 0.0386 & -5.4569 \\
\hline 171.91 & 0.0817 & 33.0158 \\
\hline 171.06 & 0.2347 & -2.0743 \\
\hline 170.32 & 0.1457 & -8.0258 \\
\hline 170.26 & 0.0096 & -13.7037 \\
\hline 169.69 & 0.1183 & -12.1544 \\
\hline 169.33 & 0.048 & -0.1258 \\
\hline 168.26 & 0.0235 & 6.1472 \\
\hline 168.15 & 0.1089 & 26.8816 \\
\hline 166.86 & 0.1039 & -36.2921 \\
\hline 166.78 & 0.1411 & -27.2709 \\
\hline 166.01 & 0.0812 & 15.2325 \\
\hline
\end{tabular}

C3

\begin{tabular}{|l|l|l|}
\hline position (nm) & Dip. Str. & R (velocity) \\
\hline $\mathbf{2 9 7 . 6 8}$ & 0.0131 & 9.9754 \\
\hline $\mathbf{2 5 1 . 4 7}$ & 2.0926 & -3.3979 \\
\hline $\mathbf{2 4 4 . 6 6}$ & 0.0097 & 9.2192 \\
\hline $\mathbf{2 4 0 . 6 9}$ & 0.1127 & 6.1324 \\
\hline $\mathbf{2 2 5 . 3 9}$ & 0.1565 & 9.4486 \\
\hline $\mathbf{2 2 1 . 6 6}$ & 3.0791 & -103.068 \\
\hline $\mathbf{2 1 2 . 0 8}$ & 1.7379 & 69.8163 \\
\hline $\mathbf{2 0 5 . 9 8}$ & 0.0349 & 11.0868 \\
\hline $\mathbf{2 0 1 . 9 4}$ & 0.0616 & 16.2358 \\
\hline $\mathbf{1 9 8 . 9}$ & 0.2287 & 6.1005 \\
\hline
\end{tabular}




\begin{tabular}{|c|c|c|}
\hline 194.16 & 0.1508 & 0.9822 \\
\hline 187.69 & 0.7728 & -32.1681 \\
\hline 186.67 & 0.123 & -3.2813 \\
\hline 185.51 & 1.1949 & 36.9779 \\
\hline 185.34 & 0.1378 & -3.6516 \\
\hline 184.87 & 0.1047 & -8.0642 \\
\hline 183.42 & 0.0448 & 0.3437 \\
\hline 183.12 & 0.1441 & -11.4806 \\
\hline 182.73 & 0.1146 & 16.8279 \\
\hline 180.38 & 0.2105 & -25.1272 \\
\hline 180.08 & 0.0882 & 3.4134 \\
\hline 179.46 & 0.1005 & -42.2455 \\
\hline 177.41 & 0.0327 & 11.2456 \\
\hline 176.12 & 0.1064 & 13.4583 \\
\hline 175.27 & 0.0237 & -13.0388 \\
\hline 174.45 & 0.1726 & 21.4054 \\
\hline 174.3 & 0.1284 & -39.4893 \\
\hline 172.81 & 0.1037 & 7.2138 \\
\hline 172.66 & 0.0207 & -3.7841 \\
\hline 172.22 & 0.0195 & -20.6983 \\
\hline 170.48 & 0.0816 & 14.8256 \\
\hline 170.38 & 0.0403 & 45.4009 \\
\hline 170.1 & 0.1965 & -17.2777 \\
\hline 169.15 & 0.0415 & -12.4724 \\
\hline 169.1 & 0.0374 & -1.5026 \\
\hline
\end{tabular}




\begin{tabular}{|l|l|l|}
\hline $\mathbf{1 6 8 . 7 4}$ & 0.0696 & -1.4512 \\
\hline $\mathbf{1 6 8 . 0 3}$ & 0.2121 & -4.884 \\
\hline $\mathbf{1 6 7 . 1 6}$ & 0.0152 & -6.5862 \\
\hline $\mathbf{1 6 6 . 0 4}$ & 0.1137 & 4.1353 \\
\hline $\mathbf{1 6 5 . 7 6}$ & 0.0933 & -8.146 \\
\hline
\end{tabular}

C4

\begin{tabular}{|l|l|l|}
\hline position (nm) & Dip. Str. & R (velocity) \\
\hline $\mathbf{2 9 7 . 5 6}$ & 0.0152 & 11.4862 \\
\hline $\mathbf{2 5 0 . 7 4}$ & 2.088 & -3.3753 \\
\hline $\mathbf{2 4 4 . 7 1}$ & 0.0107 & 9.7972 \\
\hline $\mathbf{2 4 0 . 7 6}$ & 0.1109 & 6.1035 \\
\hline $\mathbf{2 2 5 . 0 2}$ & 0.1282 & 12.881 \\
\hline $\mathbf{2 2 1 . 6 7}$ & 3.0932 & -109.051 \\
\hline $\mathbf{2 1 2 . 1}$ & 1.7376 & 70.7545 \\
\hline $\mathbf{2 0 6}$ & 0.0341 & 10.9446 \\
\hline $\mathbf{2 0 1 . 6 8}$ & 0.0817 & 16.8755 \\
\hline $\mathbf{1 9 9 . 4 5}$ & 0.2359 & 2.8996 \\
\hline $\mathbf{1 9 4 . 3 1}$ & 0.1515 & 0.5646 \\
\hline $\mathbf{1 8 7 . 7 5}$ & 0.7153 & -32.7062 \\
\hline $\mathbf{1 8 6 . 5}$ & 0.1935 & 11.508 \\
\hline $\mathbf{1 8 5 . 4}$ & 0.1178 & -7.3877 \\
\hline $\mathbf{1 8 4 . 9 8}$ & 0.3612 & 26.0033 \\
\hline $\mathbf{1 8 4 . 1}$ & 0.9028 & -7.2289 \\
\hline $\mathbf{1 8 3}$ & 0.0588 & -25.3838 \\
\hline
\end{tabular}




\begin{tabular}{|c|c|c|}
\hline 183.06 & 0.1026 & 7.2077 \\
\hline 182.46 & 0.0776 & 7.4391 \\
\hline 180.01 & 0.1545 & -2.7109 \\
\hline 179.4 & 0.3274 & -115.882 \\
\hline 179.2 & 0.1613 & 17.1858 \\
\hline 177.28 & 0.0611 & 8.2369 \\
\hline 176.15 & 0.0678 & 10.9368 \\
\hline 175.13 & 0.005 & 8.7187 \\
\hline 174.63 & 0.0614 & -0.6406 \\
\hline 174.46 & 0.1837 & 7.1254 \\
\hline 172.87 & 0.0724 & 4.601 \\
\hline 172.48 & 0.0175 & -0.7515 \\
\hline 172.01 & 0.0422 & -5.6236 \\
\hline 170.51 & 0.1298 & 24.7315 \\
\hline 170.15 & 0.1226 & -39.4955 \\
\hline 169.44 & 0.0726 & -0.2872 \\
\hline 169.12 & 0.0368 & 2.734 \\
\hline 168.81 & 0.0839 & 3.7979 \\
\hline 168.6 & 0.0771 & 0.7782 \\
\hline 167.96 & 0.1445 & 6.8075 \\
\hline 167.39 & 0.0799 & 8.9998 \\
\hline 166.8 & 0.1158 & -9.7913 \\
\hline 166.2 & 0.072 & -20.3634 \\
\hline
\end{tabular}


Table S8: ORD values at camb3lyp/Aug-cc-pVDZ/PCM for 4 lowest energy conformations of (-)$(6 R, 7 R, 8 S, 10 R, 2 ' Z)$-centratherin.

\section{Conformation}

\begin{tabular}{|c|c|c|c|c|}
\hline [Alpha] & $\mathbf{1}$ & $\mathbf{2}$ & $\mathbf{3}$ & $\mathbf{4}$ \\
\hline $\mathbf{6 3 3}$ & -39.23 & -60.48 & -37.71 & -68.55 \\
\hline $\mathbf{5 8 9}$ & -44.34 & -67.85 & -42.61 & -78.41 \\
\hline $\mathbf{5 4 6}$ & -49.87 & -75.46 & -47.84 & -89.71 \\
\hline $\mathbf{4 3 6}$ & -57.49 & -78.36 & -52.78 & -118.25 \\
\hline $\mathbf{4 0 5}$ & -46.48 & -52.65 & -38.4 & -112.51 \\
\hline $\mathbf{3 6 5}$ & 19.52 & 81.32 & 44.19 & -36.6 \\
\hline
\end{tabular}


Figure S1: Three dimensional structural views of the four lowest energy conformers of (-)$(6 R, 7 R, 8 S, 10 R, 2 ' Z)$-centratherin.

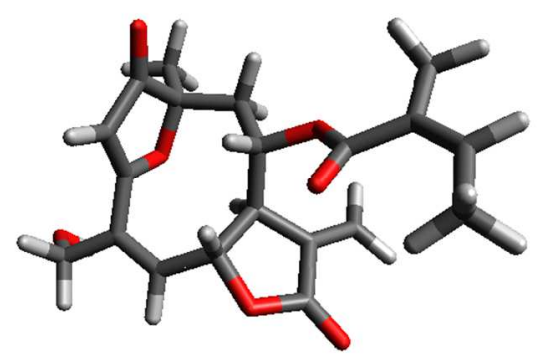

C1:

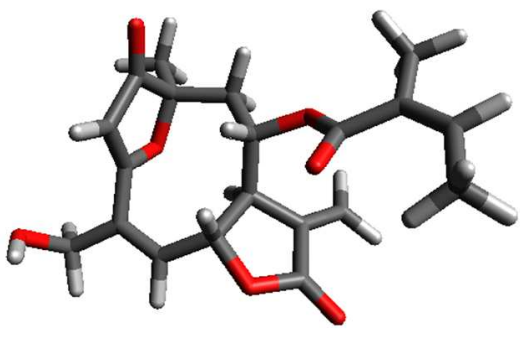

C2:

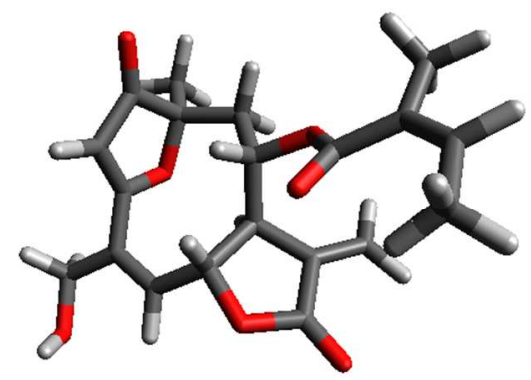

C3:

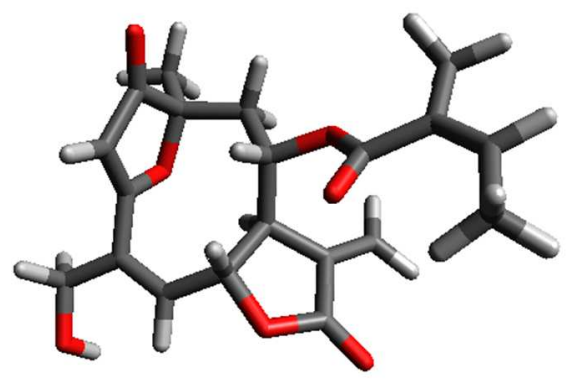

C4: 
Figure S2: Overlaid structural views of the four lowest energy conformers of (-)-(6R,7R,8S,10R,2'Z)centratherin

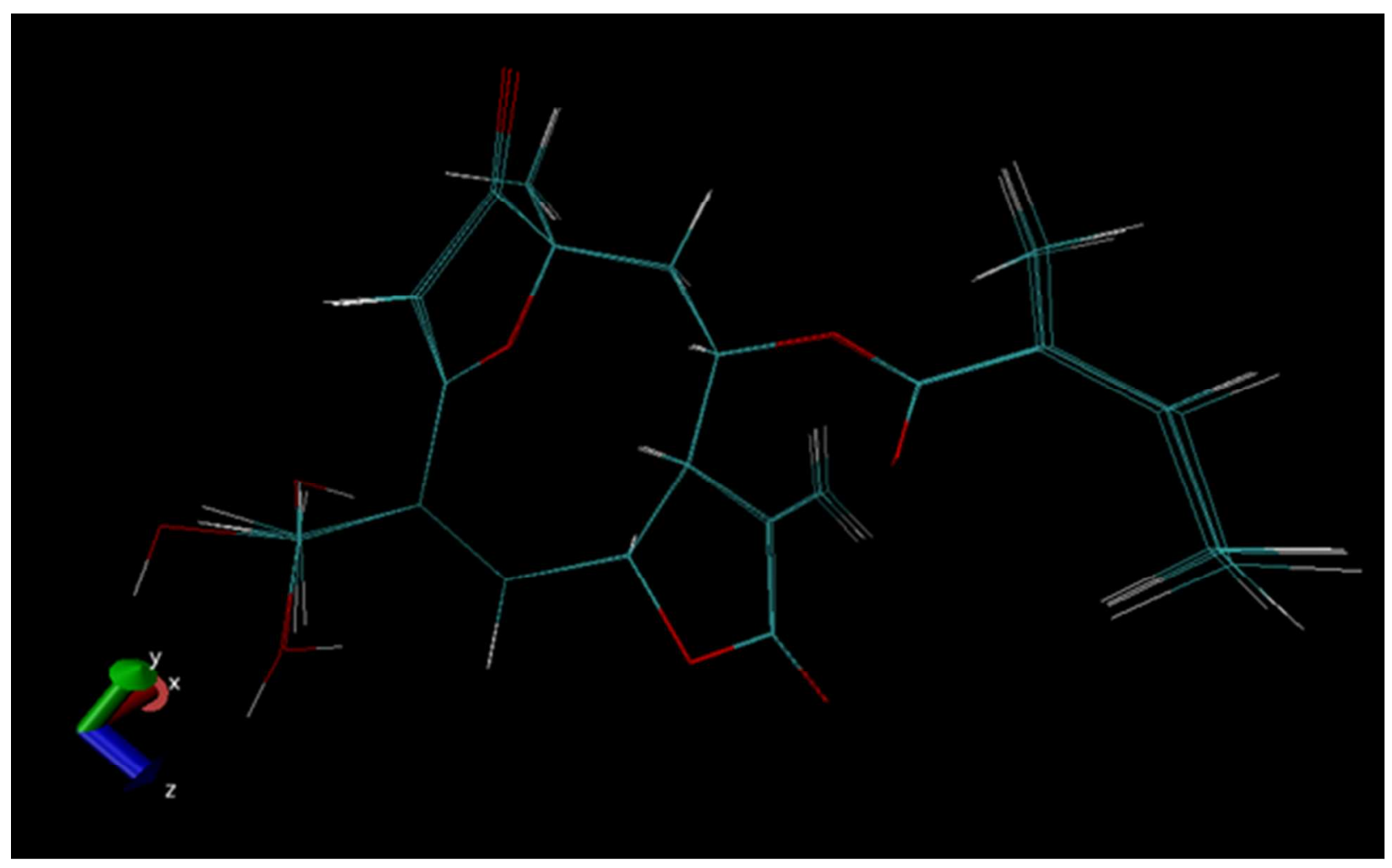


Figure S3: Vibrational Infrared absorption spectra of four lowest energy conformers of (-)$(6 R, 7 R, 8 S, 10 R, 2 ' Z)$-centratherin

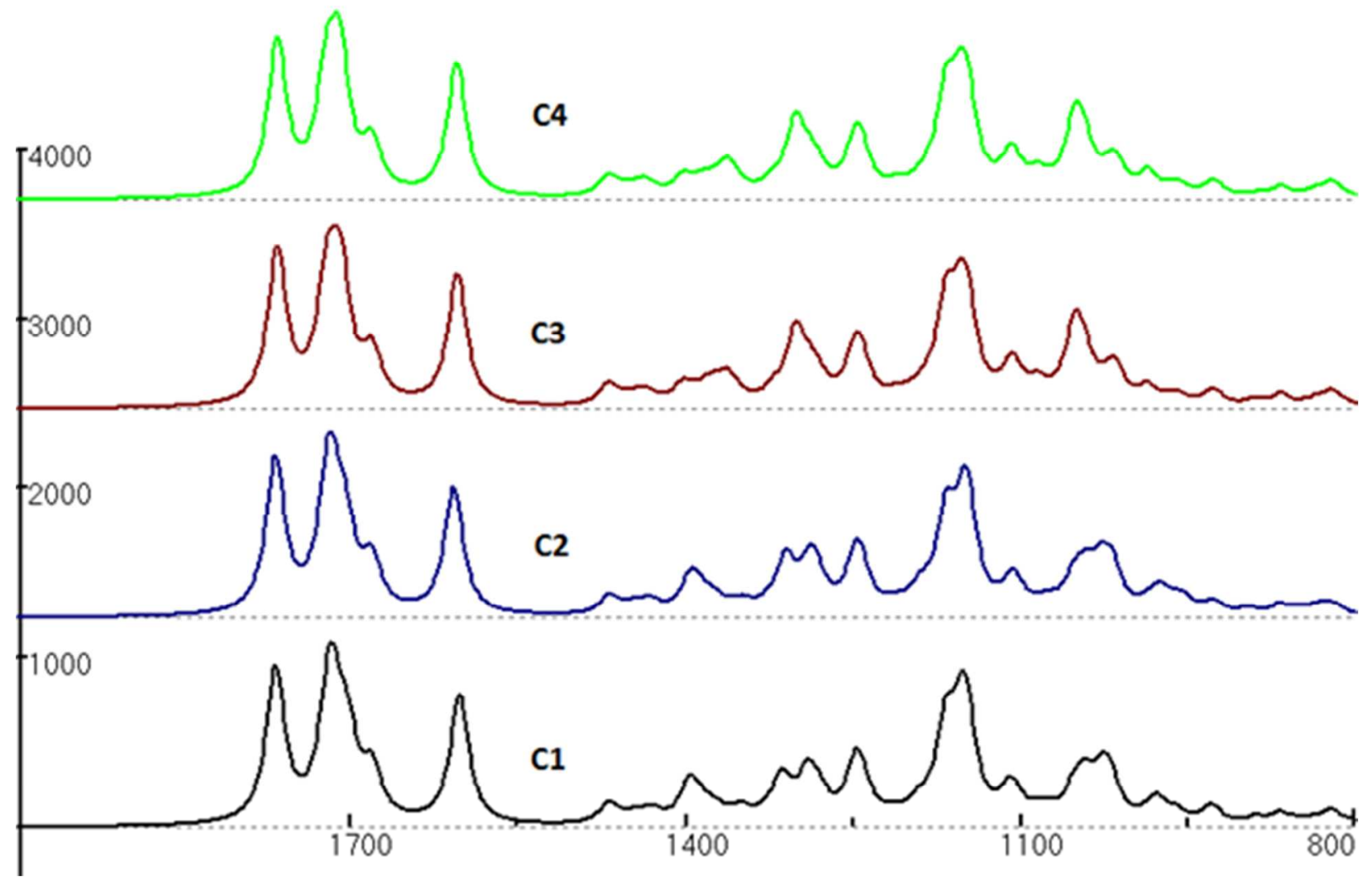


Figure S4: Vibrational circular dichroism spectra of four lowest energy conformers of (-)$(6 R, 7 R, 8 S, 10 R, 2 ' Z)$-centratherin

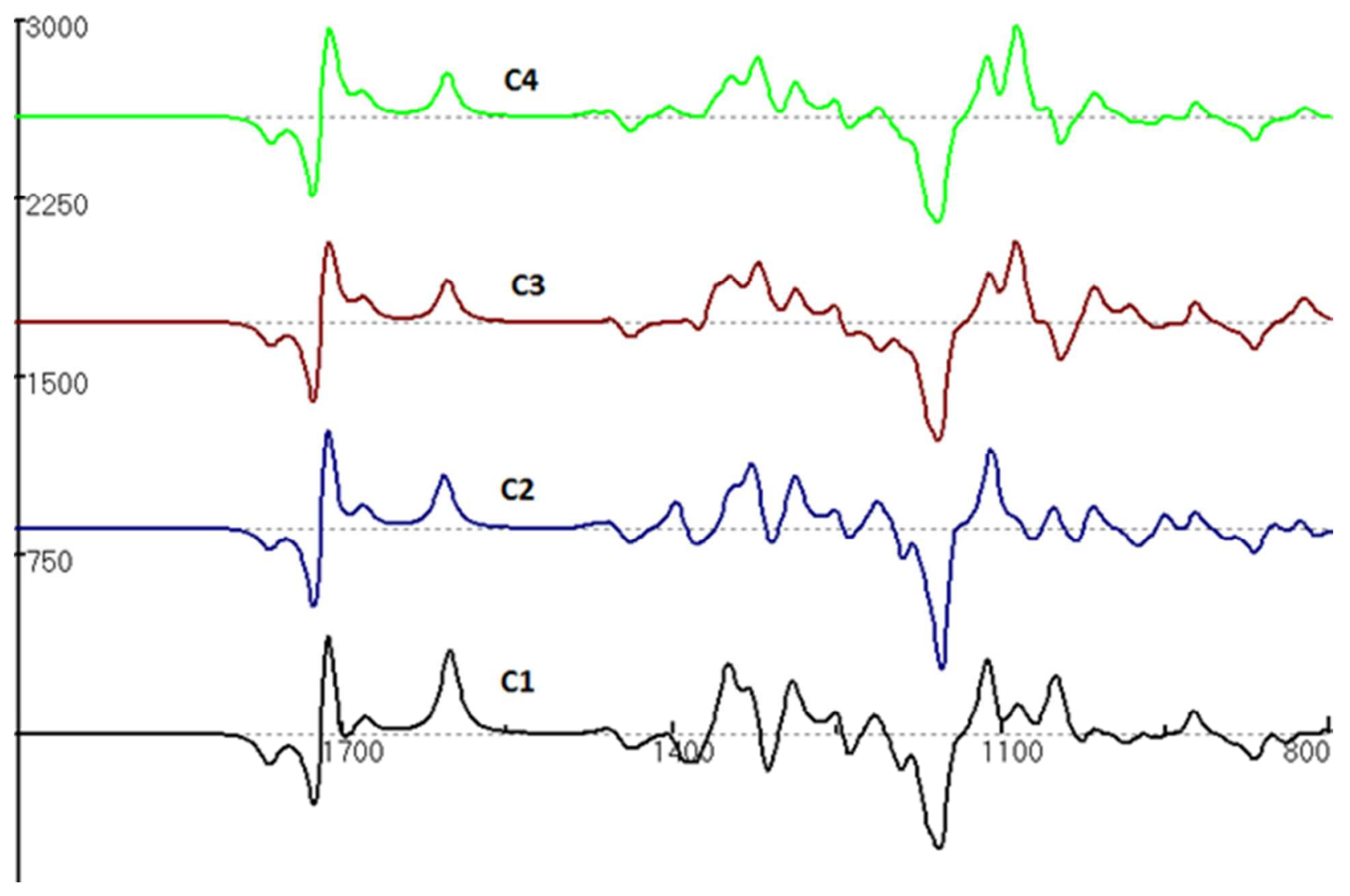




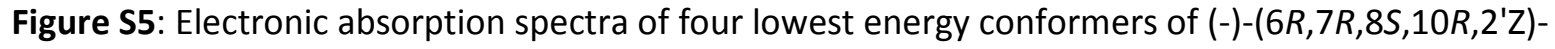
centratherin
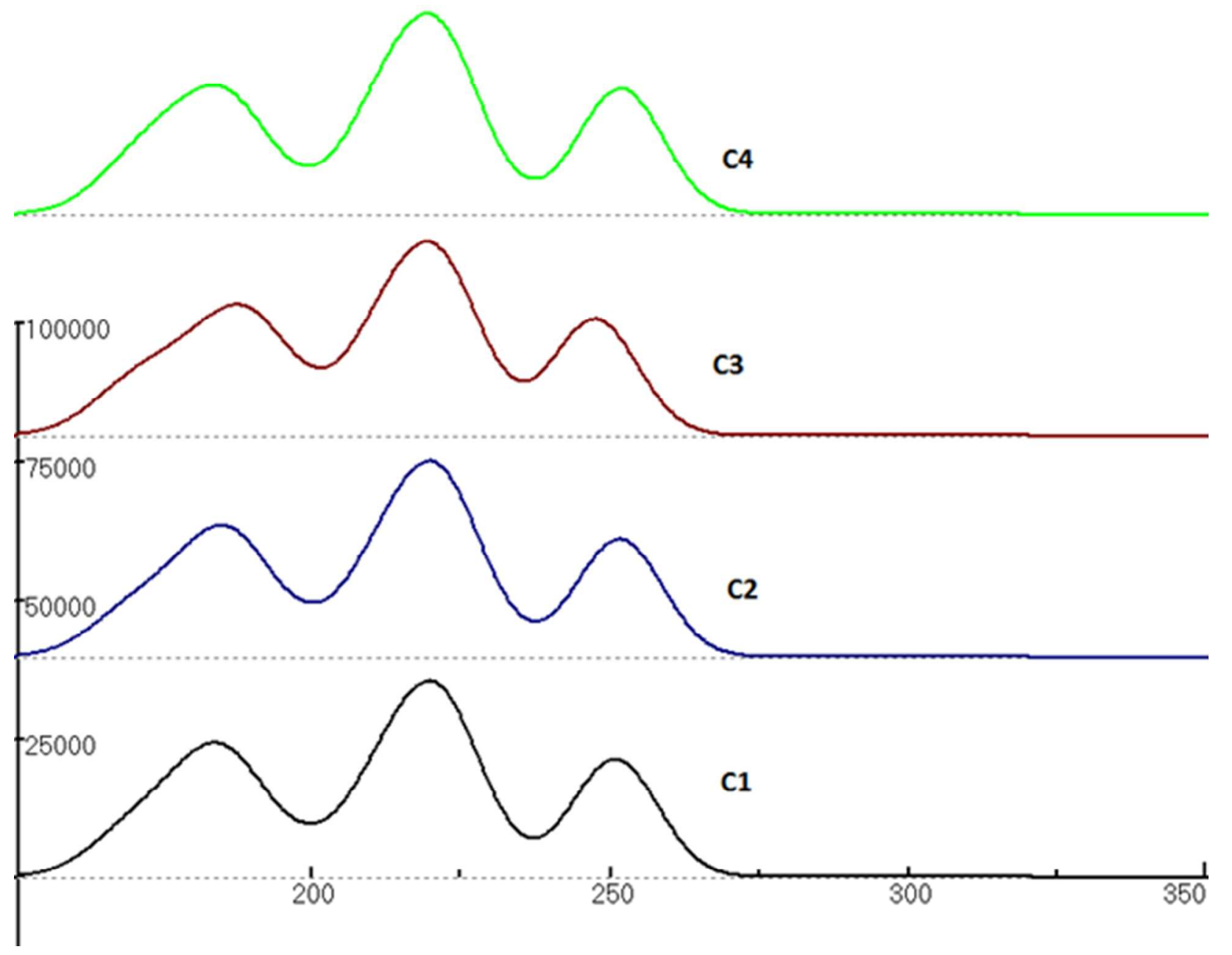
Figure S6: Electronic circular dichroism spectra of four lowest energy conformers of (-)$(6 R, 7 R, 8 S, 10 R, 2 ' Z)$-centratherin

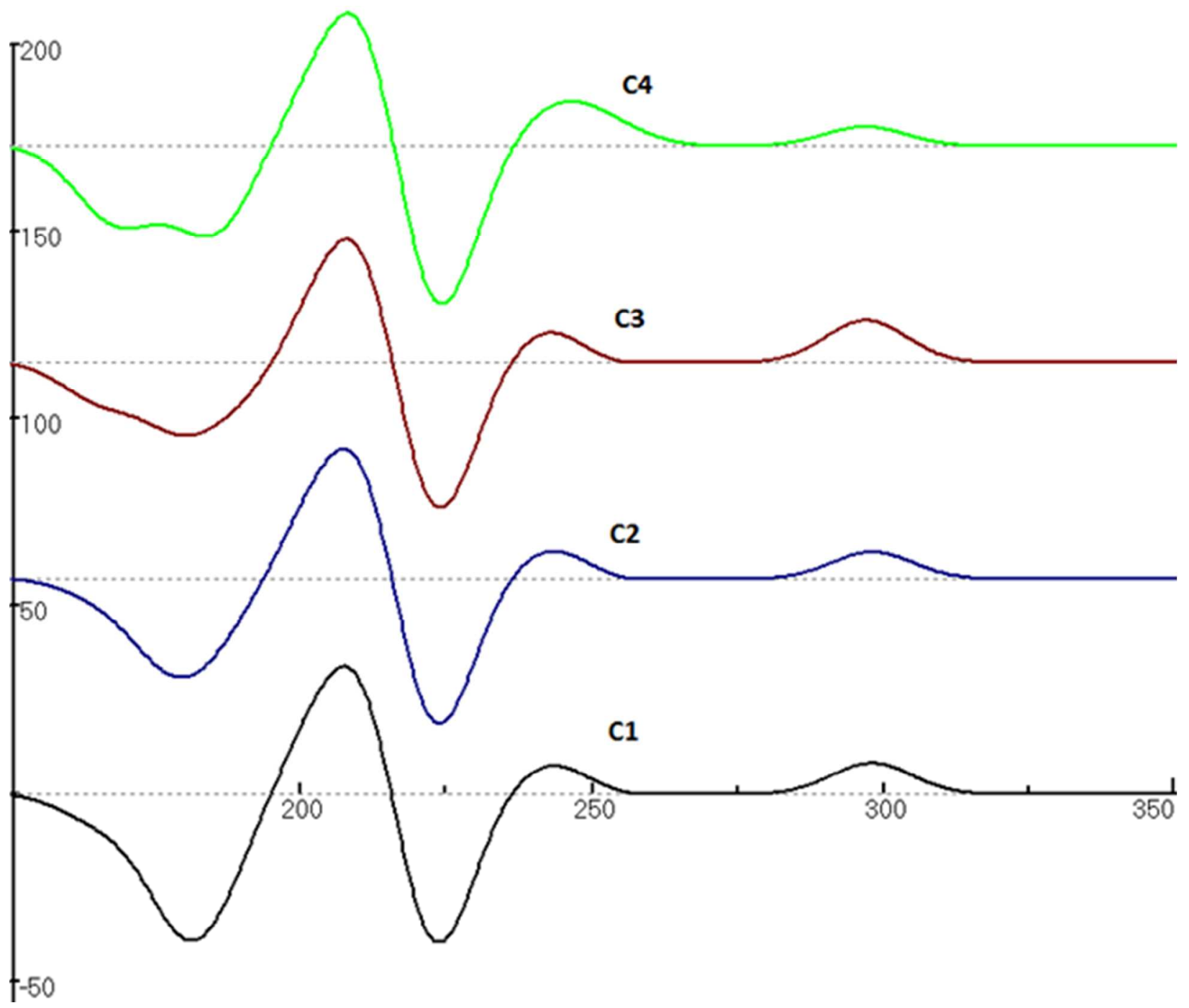


Figure S7: Predicted electronic circular dichroism spectrum (-)-(6R,7R,8S,10R,2'Z)-centratherin (Figure 1B in the main text) with rotational strengths displayed as vertical bars and its comparison to experimental spectrum (red trace).

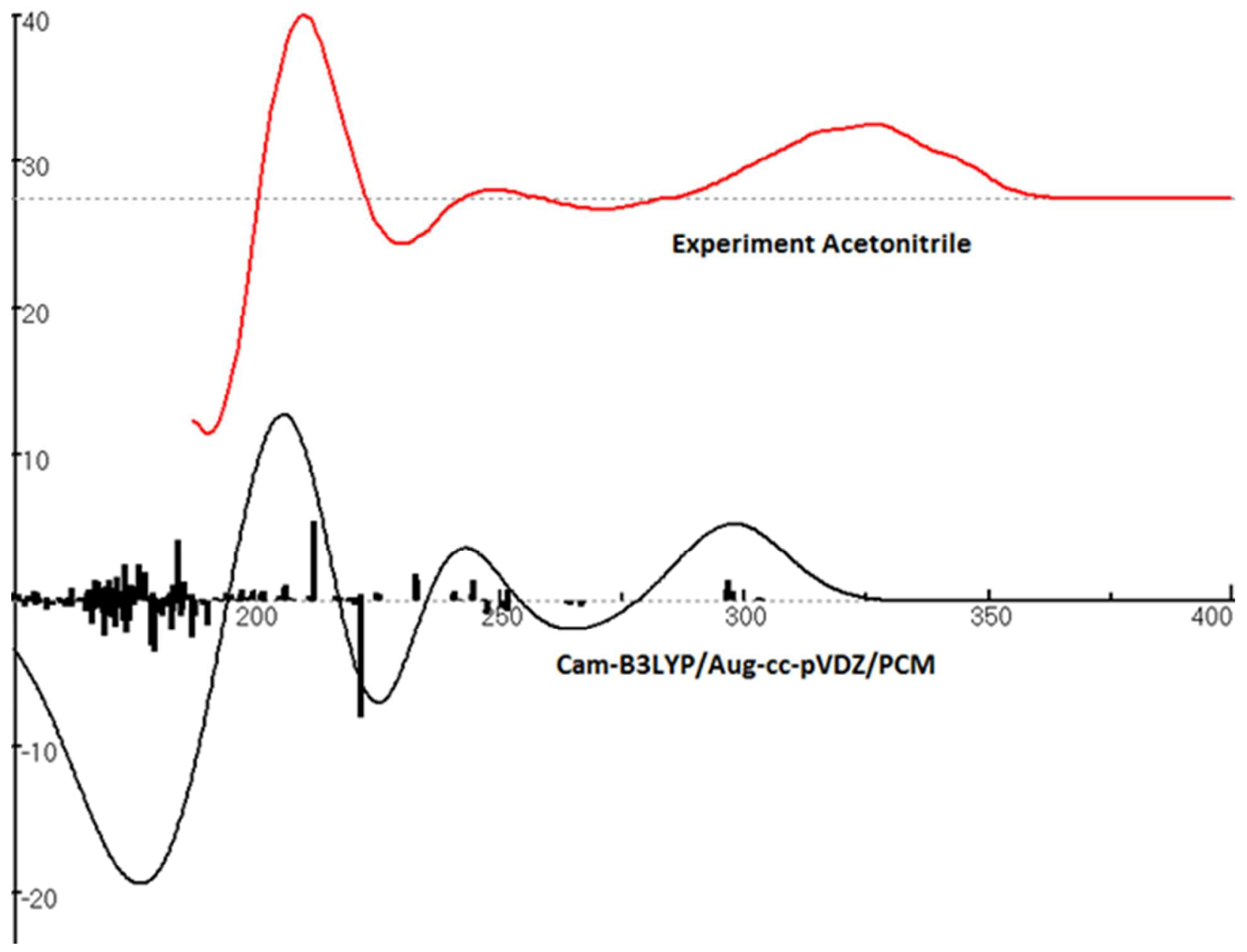

